\title{
Les 11-18 ANS ET LES BIBLIOTHËQUES MUNICIPALES
}

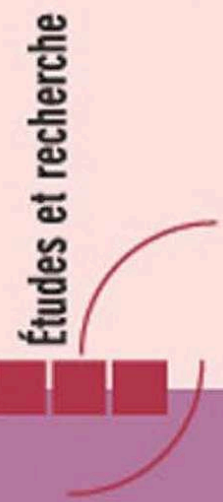

Rédigé par Virginie Repaire

(BS consultants)

et Cécile Touitou

(Tosca Consultants). avec la collaboration de Bernard Sallet et de Françoise Bettahar

(BS consultants),

septembre 2009

Bibliothèque

登 Centre

Pompidou 


\section{Les 11-18 ans et les bibliothèques municipales}

\section{Virginie Repaire et Cécile Touitou}

DOI : 10.4000/books.bibpompidou. 1024

Éditeur : Éditions de la Bibliothèque publique d'information Année d'édition : 2010

Date de mise en ligne : 17 janvier 2014

Collection : Études et recherche

ISBN électronique : 9782842461263

\section{Sbooks}

http://books.openedition.org

\section{Édition imprimée}

Nombre de pages : 37

\section{Référence électronique}

REPAIRE, Virginie; TOUITOU, Cécile. Les 17-18 ans et les bibliothèques municipales. Nouvelle édition [en ligne]. Paris : Éditions de la Bibliothèque publique d'information, 2010 (généré le 02 février 2021). Disponible sur Internet : <http://books.openedition.org/bibpompidou/1024>. ISBN : 9782842461263. DOI : https://doi.org/10.4000/books.bibpompidou.1024.

(c) Éditions de la Bibliothèque publique d'information, 2010

Conditions d'utilisation:

http://www.openedition.org/6540 


\section{Les 11-18 ans et les bibliothèques municipales}

Rédigé par Virginie Repaire (BS consultants)

et Cécile Touitou (Tosca Consultants),

avec la collaboration de Bernard Sallet

et de Françoise Bettahar (BS consultants),

septembre 2009

Bibliothèque

歰 Centre publique d'information Pompidou

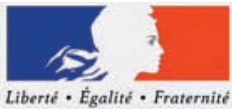

RÉPUBLIQUE FRANÇAISE

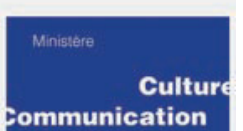


Président

du Centre Pompidou

Alain Seban

Directrice générale

du Centre Pompidou

Agnès Saal

Délégué à

l'Action culturelle

et à la Communication

Philippe Charrier

Responsable du pôle

Accueil des publics

Emmanuel Aziza

Chef du service Études

et recherche

Françoise Gaudet

Chef du service

Édition/Diffusion

Arielle Rousselle

(c) Éditions de la Bibliothèque publique d'information/Centre Pompidou, 2010.

ISBN 978-2-84246-126-3 


\section{Sommaire}

Synthèse

\section{Introduction}

Méthodologie de l'enquête

\section{Principaux résultats}

7 De l'appropriation à l'indifférence : les variations selon les caractéristiques socioculturelles et l'offre proposée par les bibliothèques

7 « Du plaisir au travail » : des usages qui évoluent au fil du temps

8 Les collégiens : un lieu « qui mélange détente - plaisir et travail »

10 Les lycéens, les étudiants : de bonnes conditions de travail

11 «Pourquoi je n'y vais plus ? Ça, c'est la question piège !» : les raisons du « décrochage »

15 « Je ne suis pas dans mon élément » : un univers étranger

16 «C'est trop calme »

17 D'autres sources d'approvisionnement

19 Des représentations qui convergent, mais des demandes contrastées

19 La maison du livre : un lieu calme et sérieux, légitimement codé

22 «C'est mystérieux les bibliothécaires » : un métier statique et ennuyeux

24 Des demandes contrastées

26 Des pistes pour rebondir

26 Première piste : replacer le public jeune au cœur de la réflexion

27 Deuxième piste : faire bouger l'offre de manière significative

28 Troisième piste : donner plus de visibilité à la bibliothèque et faire participer les jeunes

30 Quatrième piste : Faire bouger la relation avec les publics jeunes et les autres partenaires

\section{Conclusion}

Annexe

32 Annexe quantitative 


\section{Synthèse}

\section{Introduction}

À l'heure d'Internet, y a-t-il encore une place pour la bibliothèque municipale ${ }^{1}$ dans l'univers des adolescents ? Quel intérêt peut-elle présenter aux yeux de jeunes qu'on imagine volontiers faibles lecteurs, adeptes convaincus des moteurs de recherche, et virtuoses du téléchargement, légal ou illégal, de musique et de films notamment? Pourquoi certains jeunes ne viennent-ils pas ou plus dans les bibliothèques et que pourrait-on leur offrir pour qu'ils les fréquentent? Autant de questions qui préoccupent légitimement les bibliothécaires en ce début de $\mathrm{XXI}^{\mathrm{e}}$ siècle dans un contexte de baisse avérée de la lecture de livres².

À première vue, les résultats des enquêtes menées sur le sujet ne semblent pas confirmer une désertion des publics jeunes. Ainsi, d'après une enquête nationale réalisée en 2001-2002, $92 \%$ des moins de 15 ans déclaraient être allés au cours de leur vie dans une bibliothèque, et quatre sur dix l'avaient fréquentée depuis la rentrée scolaire ${ }^{3}$. Les enquêtes menées sur les plus de 15 ans relèvent de leur côté que les jeunes adultes (15-19 ans) affirment massivement avoir fréquenté une bibliothèque au cours des 12 derniers mois, plus qu'aucune autre classe d'âge ${ }^{4}$. En revanche, toutes les études, les données statistiques locales quand elles existent - et les conclusions de la présente enquête - enregistrent une désaffection progressive au cours de l'adolescence, plus marquée chez les garçons que chez les filles ${ }^{5}$. Par ailleurs, les données quantitatives ne disent pas grand-chose sur l'appropriation réelle de la bibliothèque, qu'il s'agisse de jeunes ou d'adultes - est-ce parce qu'on a mis une fois le pied dans la bibliothèque de sa ville au cours des 12 derniers mois que l'on peut être considéré comme un fréquentant ? Ce que montrent surtout ces enquêtes, c'est que les bibliothèques municipales font désormais partie du décor, si ce n'est du quotidien des Français, et en particulier des jeunes générations, public soumis à de fortes prescriptions. Mais cette familiarité reste toute relative sachant qu'elle va de pair avec une fréquentation en baisse et surtout une forte méconnaissance de l'offre réelle de ces équipements ${ }^{6}$.

Afin d'apporter de nouveaux éléments de réflexion, l'enquête réalisée en 2008 à la demande de la Direction du livre et de la lecture du ministère de la Culture et de la Communication est ciblée sur une tranche d'âge originale, celle des 11-18 ans, rarement étudiée en tant que telle. Ce groupe d'âge élargi ne peut évidemment pas être considéré comme homogène, bien au contraire. Au cours de ces quelques années, qui correspondent en gros aux études secondaires (collège et lycée), la vie de ces jeunes sera marquée par différentes étapes : la fin de l'enfance et l'entrée dans l'adolescence, qui selon les sexes et les individus se fera plus ou moins tôt ; très vite pour certains, l'entrée dans la vie professionnelle, voire dans l'âge adulte ; la disparition progressive de l'autorité parentale et de celle des prescripteurs institutionnels que sont l'école et parfois la bibliothèque; la montée en puissance du temps des copains et de la « tyrannie ${ }^{7}$ " des pairs pour des jeunes de plus en plus soumis à la pression du groupe d'amis, à l'heure où la diffusion des outils de communication instantanée impose un rythme et une disponibilité de tous les instants aux sollicitations amicales. 
L'enquête sur le rapport que les jeunes âgés de 11 à 18 ans entretiennent avec les bibliothèques municipales est à la fois une enquête de population et une enquête sur site. La phase terrain a été effectuée en 2008 sur six sites reflétant la diversité des bibliothèques françaises, l'option retenue étant d'interroger des adolescents de 11 à 18 ans vivant à proximité d'un réseau de lecture publique. L'étude comprend deux phases réalisées conjointement :

- Une première phase au cours de laquelle 175 entretiens semi-directifs ont été réalisés auprès de jeunes âgés de 11 à 18 ans, rencontrés à Lille (59), Toulouse (31), Auxerre (89), Nanterre (92), Graulhet (81) et Dinan (22), et qui fréquentent ou ne fréquentent pas (ou plus) les bibliothèques des réseaux étudiés. Les sites d'étude ont été retenus pour leur caractère à la fois « représentatif » de la diversité des communes françaises (petites, moyennes et grosses villes), et pour le caractère " contrastif » des réseaux de lecture publique proposés aux populations desservies (établissements récents ou non, types d'espaces, de collections et de services dédiés aux jeunes, politiques tarifaires, etc.) Sur chaque site, des groupes de discussion (focus groups) ont été organisés pour compléter les entretiens individuels.

- Une seconde phase au cours de laquelle près de 1200 questionnaires autoadministrés ont été recueillis auprès de fréquentants âgés de 11 à 18 ans sur les sites de Lille et Toulouse (dans les centrales et dans les annexes de ces deux réseaux).

Le travail de synthèse des résultats de l'enquête s'appuie principalement 5 sur la phase qualitative réalisée sur les 6 sites sélectionnés. L’ampleur du dispositif mis en ouvre au cours de cette phase (175 entretiens semi-directifs conduits à l'aide d'un guide d'entretien ouvert auprès de personnes dont les âges varient de 11 à 18 ans) permet toutefois de dépasser l'aspect purement exploratoire auxquelles les enquêtes qualitatives sont parfois limitées. L'enquête quantitative pour sa part n'a été conduite que sur les réseaux de Lille et Toulouse pour des raisons d'ordre pratique (une fréquentation intense étant nécessaire afin d'obtenir des taux de retour du questionnaire autoadministré et des échantillons statistiquement significatifs) ; cette phase de l'enquête avait pour principal objectif de cadrer certains ordres de grandeur (ce que font les 11-18 ans en bibliothèques, leurs profils, etc.), sachant que les résultats obtenus ne peuvent évidemment pas être généralisés systématiquement à l'ensemble des sites étudiés et que l'investigation ne porte ici que sur les fréquentants des bibliothèques. 


\section{Principaux résultats $\rightarrow$}

Il est désormais difficile, cette enquête le confirme, de rencontrer un jeune Français qui n'est pas allé au moins une fois dans sa vie dans une bibliothèque municipale, que ce soit sous la conduite de ses parents ou celle de l'école ${ }^{8}$. Par ailleurs, comme chez les adultes, l'image que ces jeunes ont de la bibliothèque est globalement positive. L'utilité de la bibliothèque fait l'objet d'un consensus assez général, même chez les non-fréquentants, lesquels admettent volontiers que la BM peut être utile : pour les autres, pour eux-mêmes plus tard, pour leurs futurs enfants...

On reste néanmoins frappé par le caractère convenu de ces représentations. La bibliothèque est toujours perçue, majoritairement, comme le lieu du livre, de l'étude, du silence... stéréotypes qui reviennent au fil des entretiens, aussi bien chez les usagers que chez les non-usagers. De même, les bibliothécaires restent assimilés à des gardiens du calme. L'enquête met en évidence, même chez ces jeunes beaucoup plus investis dans la fréquentation des bibliothèques que leurs aînés, une sorte de "familiarité distante" " déjà identifiée chez les adultes. S'il est finalement assez rare de rencontrer chez les adolescents une franche hostilité à la bibliothèque, l'attitude la plus fréquente relève surtout de l'indifférence. On "n'y pense pas ", on décroche progressivement, presque en douceur, plutôt qu'on ne rejette la bibliothèque avec virulence.

Cette indifférence, alliée à une méconnaissance profonde de ce que sont les bibliothèques municipales aujourd'hui, reste le principal problème auquel sont confrontés les bibliothécaires. Même chez les usagers les plus assidus, l'enquête révèle une appropriation relativement restreinte de la bibliothèque, limitée par exemple à l'espace jeunesse, ce qui ne facilite pas la circulation entre les 6 différentes offres susceptibles de retenir l'attention des jeunes, ni le passage de $\frac{6}{\text { Principaux résultats }}$ la section enfants à la section adultes.

De l'enfance à l'entrée dans l'âge adulte, ce passage s'accompagne le plus souvent d'un relâchement de la fréquentation - voire d'un abandon - mais aussi souvent d'un usage différent de la bibliothèque. Les usages des collégiens de 11 à 14 ans restent principalement liés à leurs loisirs, tandis que, pour les 17-18 ans enquêtés, la bibliothèque est surtout perçue comme un lieu offrant des conditions optimales pour travailler. Différents usages et différents profils d'usagers coexistent ainsi dans les établissements. La synthèse qui suit permettra d'en avoir un aperçu plus détaillé.

Les demandes qui s'expriment, liées à ces usages différenciés, sont parfois contradictoires. Dans la bibliothèque idéale décrite par les jeunes interrogés, les espaces seraient éventuellement spécialisés selon les âges, mais surtout distribués en fonction des usages et organisés selon les niveaux de bruit autorisés. Si la demande d'espaces pour travailler au calme est forte, celle de lieux plus ludiques, confortables et conviviaux, où le bruit et la conversation en groupe seraient permis, est tout aussi présente. Plus généralement, une demande de reconnaissance du public adolescent s'exprime, qui passe notamment, mais pas seulement, par une demande d'espaces dédiés.

À partir des résultats du terrain, des suggestions des adolescents interviewés et d'un état de l'art de la littérature professionnelle, l'enquête dégage des pistes d'optimisation. 


\section{De l'appropriation à l'indifférence : les variations selon les caractéristiques socioculturelles et l'offre proposée par les bibliothèques $\rightarrow$}

L'analyse des résultats a tout d'abord été centrée sur les variations des usages et des perceptions selon les âges, le sexe, certaines caractéristiques socioculturelles des jeunes interviewés, et en regard de l'offre proposée dans les six sites retenus. On peut ainsi se demander comment s'organise(nt) le(s) rapport(s) à la bibliothèque municipale et le continuum de perceptions variées qui va de la pleine appropriation des établissements et de leur offre à l'éloignement symbolique ou réel, passant par toute une gamme de relations plus ou moins distantes à la bibliothèque. Trois trajectoires type ont été analysées en fonction notamment des groupes d'âges :

- le parcours de jeunes usagers qui conservent tout au long de l'adolescence une relation de proximité avec la bibliothèque municipale, mais dont les usages évoluent au fil du temps ;

- le parcours de jeunes en situation de " décrochage " : après avoir fréquenté, parfois assidûment, la bibliothèque ${ }^{10}$ dans leur enfance, ils s'en détournent à l'adolescence ;

- et enfin, celui des adolescents qui sont les plus éloignés de l'univers des bibliothèques municipales : même s'ils ont été initiés à la bibliothèque par l'école ou leur famille, ils considèrent ce lieu comme un territoire étranger dans lequel ils n'ont pas leur place.

\section{« Du plaisir au travail » : des usages qui évoluent au fil du temps}

À l'instar de plusieurs résultats d'études portant sur les caractéristiques des usagers des bibliothèques municipales, les rencontres faites au cours 7 de cette enquête ne dérogent pas à la règle : les adolescents les plus assidus des établissements, le plus souvent inscrits et gros lecteurs, appartiennent à lindifférence généralement à des familles elles-mêmes usagers des établissements : les frères et/ou sœurs, et bien plus encore la mère, sont usagers inscrits. Leur fréquentation commence tôt, alors qu'ils étaient en maternelle ou dans les petites classes de l'enseignement élémentaire, et se poursuit tout au long de la scolarité à l'école primaire, presque sans heurt, sinon parfois avec un rythme moins soutenu. Le passage au collège, pour ces usagers convaincus, s'accompagne généralement d'un changement d'accompagnateur : quand l'établissement est géographiquement proche du lieu de vie ou des établissements scolaires, les frères et/ou sœurs et les amis prennent progressivement la place de la mère, souvent premier prescripteur ${ }^{11}$. L'enquête quantitative réalisée à Lille et à Toulouse met aussi en lumière la forte variation des accompagnants entre le début et la fin de l'adolescence, marquée par une fréquentation plus solitaire : $51 \%$ des filles âgées de 15-18 ans déclarent venir seule, contre $34 \%$ de celles de 11-14 ans ; de même $60 \%$ des garçons de 15-18 ans viennent seul, contre $44 \%$ de ceux âgés de 11-14 ans. On le voit, dans ce domaine, les effets d'âge sont plus significatifs encore que les effets de genre, même si les filles, quel que soit leur âge, favorisent particulièrement la fréquentation avec les ami(e)s. 
Les usages des collégiens de 11-14 ans inscrits restent, comme ce fut le cas lorsqu'ils étaient enfants, principalement liés à leurs loisirs. Leurs pratiques peuvent être qualifiées de " traditionnelles ${ }^{12}$ » : emprunt de livres, de CD et de temps en temps de DVD ; lecture sur place pour le plaisir, dont des BD et des mangas. Des pratiques d'autant plus traditionnelles que ces jeunes participent rarement aux animations qui leur sont proposées mais dont ils ignorent le plus souvent l'existence, de même qu'ils utilisent peu les jeux vidéos, écoutent peu la musique sur place et visionnent rarement des films, quand ces supports sont proposés par les établissements. Ces jeunes de 11-14 ans utilisent toutefois les outils informatiques, notamment dans le cadre de recherches à faire pour l'école, et cela même s'ils ont déjà un ordinateur avec un accès Internet à leur domicile. L'enquête quantitative montre en effet que l'équipement dans les foyers (voire dans la chambre) n'apparaît pas comme un frein à l'utilisation des ordinateurs des bibliothèques. Bien au contraire, les jeunes adolescents de 11-14 ans qui déclarent avoir un ordinateur dans leur chambre sont même sensiblement plus nombreux à utiliser ceux des établissements (34\% d'entre eux déclarent utiliser les ordinateurs des médiathèques pour faire des recherches sur Internet, $30 \%$ pour jouer et $25 \%$ pour utiliser Internet pour leurs loisirs, contre respectivement $27 \%, 23 \%$ et $17 \%$ parmi ceux qui n'ont pas d'ordinateur dans leur chambre). Familiers de ces outils, au point qu'on ait pu les appeler les "digital natives ", ils s'en servent aussi bien à la maison, à la bibliothèque municipale, que dans les CDI des établissements scolaires.

Quand la bibliothèque est située à proximité des collèges ou des lycées, d'équipements de loisirs (comme à Dinan ou à Nanterre), du domicile (comme dans le cas des bibliothèques de quartier), ou qu' elle est bien desservie par les transports en commun (comme la centrale de Toulouse), elle est aussi régulièrement visitée en groupe, et ce quel que soit le jour de la semaine. Il en est ainsi de la médiathèque José Cabanis à Toulouse dans laquelle se rend souvent Noémie, 13 ans, le dimanche après-midi avec ses amies :

" Elle est à côté de la gare et on peut prendre le métro pour y aller, donc c'est vachement pratique (...) si on ne sait pas quoi faire, du coup tu prends le métro, et hop, tu y es!»

L'ouverture dominicale est ainsi largement appréciée par les jeunes rencontrés soit parce que « le samedi, c’est plus le jour où tu vas aller faire les courses, tu vas faire les boutiques. Alors que le dimanche tu n'as rien à faire, tout est fermé et tu vas à la médiathèque ", explique Fanny, 13 ans, soit parce que, selon Diane du même âge, «la semaine je suis prise par mon travail et le soir je préfere me consacrer à mes devoirs. Pour ça l'ouverture du dimanche, c'est bien ».

Ces visites que l'on pourrait qualifier d'épisodiques, proches de la flânerie, sont surtout le fait des collégiens de 11-14 ans, non inscrits. Ils s'y rendent parfois comme ils se rendent en ville ${ }^{13}$ : pour se promener, se balader, voire pour y passer une après-midi entière pour jouer aux jeux vidéo ou pour " squatter les ordinateurs ", comme c'est le cas à la médiathèque José Cabanis, où les jeunes disent se sentir un peu " comme chez soi ». Déjà familiers des 
lieux, ils vont aussi spontanément à la bibliothèque pour " passer le temps", "faire un tour ", " patienter un peu " avant un cours de musique ou une activité sportive, ou attendre " au chaud » le ramassage scolaire, ou encore parce que la bibliothèque peut être considérée comme un bon prétexte pour se retrouver. Ainsi, Agathe, 14 ans, rencontrée à Graulhet explique :

«Les jeunes de mon âge, ils sont plutôt sur les bancs dehors (...) ils vont devant parce que (...) c'est un lieu normalement où on travaille, donc on dit [aux parents] : "Ouais, j'ai une recherche à faire ” et les parents ils t'emmènent! Moi, je ne l'ai jamais fait, mais je sais qu'il $y$ en a qui le font. Mon copain, tout le temps, à chaque fois qu'il y allait, c'était ça. Ça marche bien, comme c'est pour le travail, les parents ils disent tout le temps: "Oui, je t'emmène ”. "

Les collégiens qui fréquentent la bibliothèque en plus de leurs loisirs pour leur travail scolaire la perçoivent généralement comme " un lieu entre les deux : entre le plaisir et le travail ». Un lieu donc " qui mélange détente-plaisir et travail ", et davantage encore à partir de 15 ans, quand les adolescents commencent à avoir des usages plus diversifiés. Pour les lycéens inscrits - et plus encore pour les lycéennes -, souvent accaparés par leur travail scolaire, la fréquentation de la bibliothèque est aussi une occasion de sortir pour "se détendre ", "se relaxer ", " déstresser un peu ", "s'aérer l'esprit » précise Jawida, en terminale scientifique à Nanterre, ou encore de "s'évader ", comme le fait Charlène, 15 ans, inscrite dans une bibliothèque de quartier à Lille :

" J'emprunte parfois des livres (...) mais surtout je rêve, je profite du cadre, je me promène, je regarde les gens, je regarde les livres, j'écoute de la musique. »

Le temps passé à la bibliothèque est alors considéré comme un « moment de détente en dehors des cours " pour les lycéens en $1^{\text {re }}$ et terminale "parce qu'on a beaucoup de pression avec les études, ce qu'on a à faire et tout ça ", estime Florence, élève en $1^{\text {re }}$ littéraire à Toulouse.

La bibliothèque sera aussi un lieu de rencontre : on retrouve les amis, " ceux qu'on connaît déjà » mais aussi " pour voir qui c'est qu'il y a, genre un peu "meetic.fr" (...) les nouvelles têtes ", comme l'explique Chahida rencontrée à la bibliothèque centrale d'Auxerre. Ces adolescents s'y rendent tour à tour pour " décompresser ", se retrouver avec des amis pour discuter, utiliser les ordinateurs et travailler sur place, seuls ou en groupe... Ils y vont aussi pour "se renseigner sur les sorties de livres ", "faire un repérage ", " découvrir " des livres et des $\mathrm{CD}$ (comme c'est le cas à la médiathèque de Graulhet), ou encore les « tester gratuitement " avant de les acheter. Les établissements sont alors considérés comme des espaces de liberté : on est libre d'y venir quand on veut, d'y rester le temps que l'on souhaite, d'y circuler, de choisir ses propres lectures, et pour certains d'alterner travail scolaire, détente et lecture plaisir. Cette qualité différencie en particulier les bibliothèques des CDI où la fréquentation est, selon les jeunes, plus encadrée. 
Les plus grands circulent dans les différents espaces, alternant entre l'espace jeunesse (pour y prendre des livres, accompagner leurs frères et/ ou sœurs, mais aussi parfois pour y travailler dans une ambiance perçue comme " moins sérieuse »), l'espace ados (quand il existe), la salle d'études, l'espace multimédia ou la discothèque-cédéthèque... se dirigeant timidement vers un espace adultes, largement perçu comme étranger, de par son fonds, son public mais aussi son personnel qui semble parfois plus distant, ou plus "sérieux ". Une circulation d'autant plus timide, voire difficile, que ces jeunes commencent à peine à découvrir les espaces qui leur étaient jusqu'alors largement inconnus et ce, malgré une fréquentation parfois déjà ancienne et assidue - sauf dans le cas où, comme à Dinan par exemple, les collections de $\mathrm{BD}$ et de mangas sont volontairement placées à l'entrée du plateau adulte, comme une invitation à venir l'explorer ${ }^{14}$.

On constate donc une méconnaissance assez générale des espaces, des supports et des activités qu'offre la bibliothèque, parfois accentuée, comme à la bibliothèque centrale d'Auxerre, par la conception même des bâtiments qui ne facilite pas la circulation. Sans accompagnement, cette méconnaissance est susceptible de se pérenniser aux âges les plus avancés, au point que certains jeunes ignoreront encore à 16 ou 17 ans la présence de services et/ou de supports susceptibles pourtant de les intéresser, tels que l'accès à Internet. À cela s'ajoutent, selon certains, des difficultés d'accès aux services, notamment en matière d'offre informatique : les jeunes interviewés évoquent souvent un nombre de postes informatiques insuffisant, l'accès limité à Internet, ou parfois encore la nécessité d'une réservation. Insuffisante information sur cette offre, rareté de celle-ci, manque d'attention ou d'intérêt de la part des jeunes usagers, les raisons peuvent s'additionner pour expliquer ce manque d'utilisation.

Les lycéens, les étudiants : de bonnes conditions de travail

Les plus âgés, lycéens et étudiants, qui ne se considèrent plus vraiment à 17-18 ans comme des adolescents mais bien comme des jeunes adultes, perçoivent surtout la bibliothèque comme un lieu offrant de bonnes conditions de travail, loin de toute tentation de distraction. Il en est ainsi plus particulièrement à la bibliothèque Jean Lévy à Lille $e^{15}$ et à la médiathèque Pierre et Marie Curie à Nanterre, où ces jeunes de 17-18 ans viennent pour travailler sur place, faire des recherches et/ou réviser des examens, seuls ou en groupe. Loundja, 18 ans, étudiante en $1^{\text {re }}$ année de médecine se rend à la médiathèque centrale de Nanterre «toujours que pour travailler. Je n'emprunte pas de livres. Je ne suis pas inscrite, je n'ai plus de carte. J'y révise mes cours, c'est tout ». Elle apprécie l'organisation spatiale propice à la concentration :

«L'éclairage est doux. Les tables, ça va. Il y a des tables individuelles et des tables pour des groupes, c'est bien. Ce qui est bien, c'est que contrairement à la BU, les tables ne sont pas sur le passage des gens, elles sont décalées, séparées des rayonnages et du passage, donc on n'entend pas le bruit des gens. Ça dérange moins. Il y a une grande séparation entre adultes et enfants, avec l'entrée, le hall, et on n'entend rien du tout. Cela fait deux mondes différents, bien séparés. » 
Ces jeunes adultes utilisent tour à tour les espaces de travail, les places de travail, parfois Internet ou le fonds documentaire ; ponctuellement, on en rencontre qui se sont inscrits pour emprunter des documents de travail, même si les contraintes liées à l'emprunt dissuadent la majorité d'entre eux. Ils sont nombreux à regretter la fermeture des établissements le dimanche. À la recherche du calme studieux qui peut faire défaut au CDI ou au domicile, ils sont plus fréquemment non inscrits - c'est le cas de $25 \%$ des fréquentants âgés de 18 ans, contre $7 \%$ seulement des fréquentants âgés de 11 ans, selon les résultats de la phase quantitative de l'enquête menée à Lille et Toulouse. Ils ne viennent guère en ce cas à la bibliothèque que pour le travail scolaire (pour près de $66 \%$ des non inscrits, contre environ $49 \%$ des inscrits), s'y rendant plus occasionnellement que les jeunes inscrits, mais en restant parfois plusieurs heures sur place. Fréquentant les bibliothèques par choix et par commodité - et les fuyant quant ils trouvent l'ambiance encore trop bruyante - ces jeunes adultes estiment que le travail en bibliothèque favorise l'acquisition de nouvelles compétences, l'apprentissage de l'autonomie, indispensables pour les études futures. C'est particulièrement le cas de ceux dont les parents sont issus de l'immigration. Les bibliothèques municipales sont alors considérées comme une " chance " de progresser dans les études et de " construire un avenir». On peut imaginer par ailleurs que cette pratique favorise la fréquentation ultérieure des bibliothèques universitaires.

«Pourquoi je n'y vais plus ? Ça, c'est la question piège ! »:

les raisons du décrochage

11

De l'appropriation

Qu'ils soient usagers ou non, les adolescents qui ont interrompu, même à l'indifférence provisoirement, leur fréquentation sont largement représentés parmi ceux qui n'ont aucun membre de leur famille actuellement inscrit dans une bibliothèque municipale. Le plus souvent rencontrés parmi ceux qui ont eu comme premier prescripteur l'école, ils gardent en général un souvenir agréable, voire nostalgique de leur première visite, à l'instar de Mickaël, jeune collégien graulhetois de 14 ans, qui se rappelle ses premières visites à l'ancienne bibliothèque de Graulhet :

" Il y avait une personne qui lisait des contes, elle lisait des histoires et il y avait des jeux. Et puis, on pouvait aussi prendre des livres pour lire à la maison... enfin, feuilleter. C'était bien, c'était enrichissant. »

Dans leur très grande majorité, ces jeunes ont des difficultés à expliquer les raisons qui les ont conduits à ne plus fréquenter les bibliothèques. Linterruption de leur fréquentation, temporaire ou durable, semble toutefois plus facilement explicable, car sans doute plus justifiable, lorsqu' elle a eu lieu dans les petites classes de l'enseignement primaire : elle est généralement légitimée par l'absence de devoirs ou de recherches à faire pour l'école. Lorsque l'arrêt de la fréquentation a eu lieu un peu plus tard, pendant les premières années de scolarisation au collège (vers 11-13 ans), les explications données sont alors beaucoup plus confuses. Les adolescents évoquent généralement 
un « manque de temps ", ou un "manque d'intérêt ", largement exprimé par "plus d'envie » ou " parce que je n'y pensais plus ${ }^{16}$ ", comme le fait Antony, 14 ans, rencontré à Toulouse :

"C'est pas ce qui me traverse l'esprit... quand je m'ennuie, je vais pas me dire "tiens je vais aller à la bibliothèque". J’y pense même pas... Je vais sortir, faire du vélo, du sport. "

Ces raisons apparaissent le plus souvent comme des sortes de " valises " pouvant contenir des éléments très divers, qui semblent en fait se conjuguer : le temps que les jeunes adolescents consacrent à leurs nouvelles activités, à leurs loisirs, la charge importante du travail scolaire... mais ils évoquent aussi, de manière quasi systématique - et souvent du bout des lèvres, un désintérêt pour la lecture, comme le fait Marie 14 ans, ancienne usager de la bibliothèque Saint-Siméon à Auxerre :

" J'ai arrêté d’y aller quand je suis passée au collège... Je n’avais plus envie... J'ai commencé à m’investir dans la gym... Maintenant Je n’ai vraiment plus de temps. Le mercredi je suis à l'AJA Gym toute l'après midi ; j'entraîne des petits. Je m’entrâne, j’ai mes devoirs à faire... La bibliothèque j’y pense pas forcément et j'ai pas forcément l'envie non plus parce que j'ai peu de temps (...) J'ai lu beaucoup pendant toute la primaire. Je lis moins maintenant, seulement de temps en temps, le week-end, pendant les vacances. »

La pratique de la lecture est alors remplacée par d'autres activités : être dehors avec les amis, écouter de la musique et/ou jouer d'un instrument, utiliser l'ordinateur, regarder la télévision et/ou des DVD, pratiquer une activité sportive, jouer à des jeux vidéos, faire les magasins... ou tout simplement « trâner » ou « rester à la maison ", diront certains. De nouveaux centres d'intérêt donc qui semblent éloigner les jeunes de 11-13 ans de la lecture et des bibliothèques, tous deux considérés sans doute comme peu valorisants aux yeux des amis, comme le laisse supposer Tamara, rencontrée à Lille :

" Je n'y suis plus trop allée vers mes 12 ans, j'étais en $5^{e}$, là c'était... je voulais faire ma grande et c'était la période où j'étais influencée quand même et donc là je ne lisais plus. J'y allais plus du tout parce que mes nouveaux loisirs c'était la musique, mes copines.»

Des centres d'intérêt recherchés à l'extérieur des bibliothèques par choix, mais aussi sans doute aussi par méconnaissance de l'offre proposée par les établissements (nombreux sont ceux à ne pas connaître l'espace multimédia ou l'offre proposée en matière de musique), ou encore par simple indifférence, ce qui les pousse à dire que cela ne leur vient même pas à l'idée.

Lorsque l'arrêt de la fréquentation se fait vers la fin du collège (vers 14-15 ans), là encore les explications prennent l'allure d'un pêle-mêle :

"Jy pense pas », «j’ai grandi », «j’ai plus envie», «j’ai Internet », «c'est pas pour les jeunes de mon âge», «ça ne minntéresse plus», «jelis peu », «jai pas le temps», «je préfere faire d’autres choses », «j’en n’ai pas besoin », «le CDI ça suffit ». 
C'est parmi ces adolescents que la présence d'Internet à la maison est parfois citée comme une des raisons d'arrêt de la fréquentation, notamment par les faibles lecteurs ; à l'instar de Mickaël, 14 ans, qui pourrait en être le porte-parole :

«Pourquoi je n'y vais plus ? Ça c'est la question piège ! Pas le temps peut-être, et puis bon, si, je crois que les moyens... Il y a eu Internet qui est arrivé et puis bon, pour faire comme des recherches... on n'aurait pas eu Internet peut-être qu’on serait allé à la médiathèque pour feuilleter des livres, des encyclopédies... que là, Internet, enfin c'est un peu la révolution, on va dire (...) ça remplace un peu la médiathèque. Par exemple, si on cherche des textes, on les tape et on peut les trouver. C'est pareil, la médiathèque, pour la musique, on peut l'avoir sur les ordinateurs. Pour moi, c'est un peu ça qui a tué la médiathèque. »

Quelles que soient les raisons évoquées, on observe une nouvelle fois que l'interruption provisoire ou plus prolongée de leur fréquentation apparaît étroitement liée avec un éloignement, lui aussi provisoire ou plus définitif, de la pratique de la lecture. La bibliothèque représentant avant tout le lieu du livre, et peut-être plus spécifiquement le lieu de la lecture de fiction, elle est alors presque logiquement délaissée par les faibles lecteurs, mais aussi par tous ceux qui estiment avoir des lectures moins nobles (comme les BD qu'ils ne considèrent pas comme des "vrais livres").

Chez les lycéens, la charge de travail qui laisse peu de disponibilité semble plus systématiquement se conjuguer avec la fréquentation d'autres établissements qui s'avère plus « rentable " pour le travail scolaire ; notamment les CDI des lycées, qui, même s'ils ne sont pas pour autant préférés, sont jugés plus " pratiques " : on peut s'y rendre entre deux heures de cours, au moment de la permanence, ils offrent un choix de documents plus important pour le travail scolaire. Les centres de documentation prennent alors le relais en matière de recherches pour le travail scolaire : les jeunes s'y rendent pour travailler sur place, parfois avec leurs professeurs, mais le plus souvent seuls ou en groupe ; ils y font tour à tour des recherches pour préparer des exposés, des exercices sur les sites mis en ligne par les collèges, ils utilisent Internet, et se renseignent sur les orientations ou les métiers. Un relais en matière de recherches scolaires, mais pas seulement, puisque beaucoup d'entre eux s'y rendent aussi pour discuter entre amis, y retrouver les anciens camarades qui ne sont plus dans la même classe, et pour leurs loisirs : lire des magazines mis à leur disposition, ou encore emprunter des $\mathrm{BD}$ ou des mangas quand les établissements le permettent. Certains jeunes considèrent ainsi que "le CDI c'est une médiathèque dans un lycée, sauf qu'il n’y a pas les CD ». D'autres préferent des établissements plus « grands » et quilils jugent plus propices au travail scolaire en raison de leur ambiance, de leurs publics et de leurs fonds, comme, en région parisienne, la Bibliothèque publique d'information du Centre Pompidou (Bpi).

Le manque de temps, cité par les jeunes adultes amateurs de lecture, s'associe avec un approvisionnement en livres plus diversifié (achat, prêt, emprunts au CDI ou dans les BU). Le poids du travail scolaire se reflète également dans les genres de livres lus, surtout liés aux loisirs au début de 
la période du collège (livres en série, $\mathrm{BD}$, récits de vie) et de plus en plus imposés par les études (théâtre, classiques, documentaires) en fin de collège ou au lycée, comme le révèlent les résultats de l'enquête quantitative menée auprès des usagers des réseaux de Lille et Toulouse. Les adolescents encore amateurs de lecture d'œuvres de fiction (souvent des filles), plus critiques à l'égard des bibliothèques autrefois fréquentées, évoquent pour leur part un « manque d'intérêt » ou "d'envie », le plus souvent associé à des éléments constitutifs aux établissements : éloignement géographique ou localisation (à Graulhet notamment où la bibliothèque est éloignée du centre-ville), horaires d'ouverture en inadéquation avec les emplois du temps scolaires, manque de nouveautés et/ou de diversité du fonds (livres fiction, BD), manque de suivi des séries, manque de lisibilité dans le classement des documents, mauvais état de certains documents, contraintes liées à l'emprunt dont les délais imposés sont considérés comme " trop courts», indisponibilité des documents..., ou encore parfois un manque de conseil prodigué par le personnel ou la difficulté à solliciter les bibliothécaires. Les critiques des amateurs de musique sur le fonds musical se font dans les mêmes termes : manque de choix, $C D$ abîmés ou déjà empruntés... mais ils semblent aussi particulièrement sensibles à la possibilité plus ou moins grande de copier les CD empruntés.

Toutes ces raisons ne sont quasiment jamais citées comme des raisons principales d'arrêt de la fréquentation, cependant elles semblent en constituer des éléments parfois déterminants, car elles représentent autant d'obstacles dans la découverte de nouvelles lectures pour le plaisir. Ces lecteurs trouvent alors, et très jeunes, de nouvelles sources d'approvisionnement pour leurs documents : bibliothèque familiale, prêt par des amis, achat et lecture sur place dans les grands magasins, CDI, et pour les amateurs de musique, CD gravés et/ou musiques téléchargées sur Internet. Ceux qui préferent posséder leurs propres livres, parfois pour les garder " en souvenir », lire les livres à leur " rythme " sans être " obligé de les rendre à un moment précis ", ou " relire une page » de temps en temps, abandonnent les bibliothèques au profit des grandes surfaces culturelles, parfois mieux situées, mais considérées surtout comme mieux approvisionnées.

Ces adolescents considèrent toutefois que les bibliothèques municipales pourraient leur être utiles plus tard, au cours de leurs études : au lycée pour les collégiens, à la préparation du baccalauréat et aux études supérieures pour les lycéens. Certains considèrent alors l'existence des bibliothèques comme sécurisante : "C'est comme un filet. Si le funambule tombe, il a un filet et il se rattrape et si moi il me manque un livre et je ne le trouve pas du tout, j’ai toujours un filet dans lequel je peux aller pour poser la question ", estime Killian, âgé de 18 ans. Cette possible reprise de la fréquentation est en fait rarement refusée a priori, mais toujours évoquée en regard de la nécessité des études, "quand les études commenceront à devenir plus sérieuses ", indiquent certains collégiens. Elle reste en effet étroitement liée à la contrainte scolaire (les jeunes adultes parlent le plus souvent en termes de "besoin ", d' "utilité » et d'obligation : «si je suis obligé... j'irais »), et n'est presque jamais imaginée en termes de plaisir et/ou de loisirs. Dans ces conditions, la bibliothèque est alors souvent considérée au même titre que les centres de documentation. Uniquement envisagée pour le travail scolaire, elle peut donc être rapidement mise en concurrence avec des établissements plus importants, 
notamment en région parisienne. Une concurrence qui peut là encore se faire avec Internet puisque, on l'a dit, l'utilisation de ce média est largement citée par les adolescents rencontrés, et cela quel que soit leur âge.

\section{« Je ne suis pas dans mon élément » : un univers étranger}

À première vue, les adolescents les plus éloignés des bibliothèques municipales sont souvent moins étrangers à cet univers qu'il n'y paraît : nombreux sont ceux qui s'y sont rendus occasionnellement, soit dans leur enfance, soit il y a peu de temps avec un ami un peu par hasard à la sortie de l'école, mais ces visites épisodiques ne leur permettent pas de donner du sens à leur démarche et par conséquent d'inscrire durablement les bibliothèques dans leur quotidien.

"La première fois c'était en $5^{\mathrm{e}}$, j'y suis allé pour faire ma carte, c'était avec ma mère et ma petite sœur. Ma mère m'a un peu obligé d'y aller ; elle voulait qu'on emprunte des livres pour qu'on commence à étudier chez nous (...) La première fois, il n'y avait pas d'animation, il n'y avait rien, c'était trop tranquille, le silence. Très peu de monde. J'ai eu une impression de vide, je ne savais pas quoi faire, où aller, avec qui. » Hosni, 16 ans.

On notera également que certains jeunes rencontrés dans le cadre de cette enquête, en plus du sentiment d'étrangeté déjà évoqué, font part de leur désorientation face aux bibliothèques, voire de leur crainte : on ne sait pas où aller, comment faire, à qui s'adresser ; on n'envisage pas de prendre le risque d'emprunter des documents de peur de les abîmer et surtout de se faire rappeler à l'ordre par le personnel à ce sujet. Deux mondes sont alors confrontés, deux univers qui ne possèdent pas les mêmes normes ni les mêmes valeurs. "Par exemple, ici, on a un langage qui va choquer certaines personnes, alors qu'avec d'autres ça passe. Honnêtement, à la médiathèque ça va pas les choquer, parce que choquer c'est un grand mot, mais ça va pas tellement passer ", analysera ainsi Fahim, non sans faire preuve pour le coup de mesure et justesse dans son expression.

La plupart du temps, les adolescents rencontrés au cours de cette enquête n'apparaissent pourtant pas véritablement hostiles aux bibliothèques ; ils instaurent simplement un rapport distancié face une offre qui, semble-t-il, ne les concerne pas, à l'image d'un jeune homme de 17 ans rencontré à Graulhet, qui considère presque naturellement que « il y en a qui y vont et d'autres qui n'y vont pas, c'est une question de choix en fait ». Un libre choix qui laisse entrevoir un certain relativisme et qui illustre particulièrement bien l'attitude générale des adolescents les plus éloignés, réellement ou symboliquement, des établissements : une attitude empreinte d'une indifférence largement assumée face à un univers qui leur est étranger, qui ne les intéresse pas, ou dont ils estiment ne pas avoir besoin. Cette indifférence apparaît aussi avec force dans la difficulté qu'éprouvent certains à se projeter dans un idéal de bibliothèque, exprimée par Stéphanie, jeune lilloise de 13 ans, qui affirme sans complexe : 
"Une bibliothèque idéale ? Y en pas! C'est soit on aime, soit on n'aime pas, c'est tout. Non, y en a pas!»

Ou encore Manon, 14 ans, elle aussi interviewée à Lille, qui semble se faire l'écho d'un grand nombre d'adolescents pour qui la bibliothèque reste encore largement associée aux livres :

«Déjà des livres, c'est une bibliothèque, mais après je ne sais pas!»

À l'image des anciens usagers assidus qui ont " décroché ", ces adolescents semblent avoir du mal à expliquer les raisons de leur non-fréquentation. Ils paraissent toutefois avoir moins de pudeur pour tenter de mettre en mots certaines des raisons qui pourraient l'expliquer : « je n'y pense pas », "ça ne m’intéresse pas ", "personne de la famille n'y va ", "aucune de mes copines ne va à la médiathèque ", "j'en n'ai pas d'utilité pour l'école ", ou encore des raisons liées au coût ou à la lourdeur administrative de l'inscription, ainsi qu’à la peur d'endommager les documents empruntés. À l'inverse de ceux qui ont longtemps fréquenté les établissements, ils reconnaissent aussi beaucoup plus facilement, sans gêne apparente, leur désintérêt pour la lecture, l'évoquant même très largement comme la raison principale de leur non fréquentation. Ce manque d'intérêt pour la lecture est souvent expliqué en termes de difficultés de concentration, en particulier par les plus jeunes, et à mettre en relation avec des difficultés scolaires.

L'ambiance calme des établissements est aussi parfois évoquée comme une raison principale de non fréquentation, comme le fait Sadika, lycéenne nanterroise âgée de 16 ans :

" Je ne viens plus depuis la $5^{\mathrm{e}}$ ou la $4^{\mathrm{e}}$. C'est trop calme. Il n'y a pas un seul bruit. Je ne supporte pas quand c'est trop calme. Je ne peux pas travailler dans le silence. Je sais qu'il ne faut pas qu'il y ait du bruit, mais je n'aime pas. Je préfere faire mes devoirs chez moi avec la télé ou avec la musique. »

Les adolescents de 15-18 ans, et peut-être plus encore les garçons non lecteurs, établissent même un lien assez direct entre le goût pour la lecture et le silence imposé dans les bibliothèques : " Je viens pas parce que je n’aime pas les endroits trop calmes et à la bibliothèque il faut être calme et j'aime pas trop lire (...) $j$ 'aime pas beaucoup aller dans les bibliothèques parce que c'est trop calme et il n'y a que des livres (...) je ne suis pas dans mon élément ", estime ainsi Ruddy en CAP de cuisine à Nanterre. Ce sentiment d'étrangeté est évidemment renforcé par la méconnaissance de l'offre actuelle des bibliothèques :

«Il y a des livres ou des romans, peut-être des documentaires, mais pas sûr. Au CDI non plus (...) À la bibliothèque, peut-être des BD, mais pas beaucoup. Peut-être des policiers (...) Je ne sais pas s'il y a 
des ordinateurs, mais je pense pas (...) Des livres (oui), des CD, je pense pas. " Céline, 13 ans.

Le plus souvent réfractaires à cette ambiance calme, perçue comme contraignante, ils la comparent alors aux contraintes imposées par l'école, préférant fréquenter d'autres établissements de la ville, où les règles seraient moins strictes (cyberbase, MJC, maison de quartier...). À l'image notamment de Fahim, jeune homme de 17 ans, apprenti électricien vivant à Graulhet :

"À la médiathèque, le souci il est là, c'est qu'on a l'impression que la médiathèque c'est comme l'école. On a l'impression de quitter l'école pour aller à l'école, quoi, ça sert pas à grand-chose. C'est ça, quand tu es là-bas, ils te disent "ne fais pas de bruit et cætera". Donc voilà, trop de restriction, ça ne donne pas forcément envie d'y aller, quoi (...) Alors qu’à la cyberbase, on sait être calme tant qu'ils ne nous disent rien, en fait. Tant qu'ils ne nous interdisent pas, on ne va pas le faire. C'est un cercle vicieux, en fait. En général c'est ça partout. Plus il y a de règle et plus... pour pas être vulgaire... on fait d'importe quoi. »

Un rejet du silence imposé dans les bibliothèques, souvent associé, comme on l'a vu, à un désintérêt pour la lecture et qui révèle que ces adolescents perçoivent les bibliothèques comme un lieu mortifere et contraignant, quasi exclusivement dédié au livre et réservé aux amateurs de lecture, plaisir qu'ils déclarent fermement ne pas partager.

Les adolescents amateurs de lectures, mais éloignés des bibliothèques se disent, quant à eux, assez réfractaires à l'emprunt, qu'il se fasse à la bibliothèque municipale, au CDI ou auprès d'amis, de peur d'abîmer les documents ou de ne pas pouvoir les consulter quand bon leur semble. Les jeunes filles, qui aiment lire en particulier les romans qui dépeignent les difficultés rencontrées par les jeunes de leur âge, supposent aussi ne pas pouvoir trouver ce genre de livres dans les bibliothèques qu'elles fréquentent occasionnellement pour leur travail scolaire, considérant qu'il ne correspond pas au «style de la médiathèque ». Quel que soit leur âge, ces amateurs de lecture ont des lieux d'approvisionnement en livres assez diversifiés (tout d'abord l'achat, la bibliothèque familiale quand les parents sont eux-mêmes lecteurs, et parfois le prêt - plus souvent rencontré parmi les filles). Bien qu'ils associent principalement, voire uniquement, la bibliothèque au livre, ils estiment ne pas avoir de raison de s'y rendre, achetant généralement leurs propres livres (souvent par l'intermédiaire de leurs parents), parfois dans des grands magasins situés non loin du domicile familial.

Le parallèle entre bibliothèques et grandes surfaces généralistes ou magasins spécialisés (notamment la Fnac, France Loisirs ou les magasins réputés des villes, comme le Furet du Nord à Lille) est donc souvent fait : les magasins sont plus attractifs et colorés, ils bénéficient parfois d'une ambiance sonore, et sont souvent plus spacieux et plus lumineux. Ils proposent un plus vaste choix de livres pour le plaisir destinés à leur âge, mieux présentés, plus de 
nouveautés, plus de documents variés : livres de toutes sortes, séries de BD, et $\mathrm{CD}$ « à la mode » ou au contraire musiques « cultes». Les grandes enseignes de magasin sont fréquentées en famille ou entre amis, par plaisir, et servent à s'approvisionner en livres, $\mathrm{BD}$, mangas, qu'on lira un peu sur place, et de temps à autres en musique, quand ils sont certains que le CD dans son entier, et pas seulement un morceau de musique, leur plaît. Les adolescents s'y sentent "plus libres " : libres d'y circuler, d'y parler, d'alterner recherche de livres pour le plaisir, lecture sur place et écoute de la musique sur les bornes prévues à cet effet. Ils estiment par ailleurs que les vendeurs-libraires sont plus compétents pour les conseiller en fonction de l'actualité ou de leurs goûts. Les adolescents y découvrent aussi des livres qu'ils ne connaissaient pas $^{17}$ et trouvent facilement les nouveautés dont ils ont entendu parler (soit par des amis, via la radio ou des sites de musique en ligne), notamment en matière de musique.

Mais la musique est toutefois très majoritairement écoutée et/ou téléchargée sur l'ordinateur, depuis Internet (où l'on visionne aussi volontiers les clips vidéo). Cette pratique est très largement citée par les adolescents rencontrés ${ }^{18}$, qu'ils fréquentent ou non les bibliothèques municipales. Le téléchargement, qui concerne la musique, mais aussi les films, est perçu comme une pratique peu coûteuse, légitimée par la présence de sites légaux et par la récente "starification " de nouveaux artistes, qui se sont fait connaittre en diffusant leurs chansons auprès des internautes. Quand les adolescents ne possèdent pas eux-mêmes un accès internet à domicile, ils passent généralement (et semble-t-il sans aucune difficulté) par l'intermédiaire d'ami(e), de grand(es) frère(s) et/ou sœur(s), ou des parents (qui en disposent souvent au travail) pour copier leurs musiques préférées sur des clés USB, avant de les transférer sur leur lecteur numérique.

En matière de travail scolaire, ces adolescents fréquentent éventuellement les salles d'étude ou les centres de documentation de leur établissement scolaire - sans pour autant apprécier ces lieux -, mais le plus souvent en complément, là encore, d'Internet et de nombreux sites généralistes ou plus spécialisés. L'utilisation d'Internet pour la recherche se fait presque automatiquement, car "plus rapide", "plus vaste ", "plus pratique ", et "sans se déplacer "... souvent sans réfléchir (ni même à la fiabilité des informations qu'ils peuvent y trouver : « Direct, sur Internet, je ne cherche pas à comprendre!»), ou d'autres fois, de façon plus raisonnée et pragmatique, en particulier chez les jeunes adultes, qui utilisent Internet en complément de documentaires et des encyclopédies qu'ils possèdent ou qu'ils consultent au CDI. L'utilisation d'Internet pour le travail scolaire semble d'autant plus facilitée, et cela dès le plus jeune âge, que les adolescents passent beaucoup de temps sur les ordinateurs et qu'ils en ont des pratiques très diversifiées : MSN, chat, consultation et/ou création de blogs, jeux seuls ou en réseaux, recherches diverses pour le plaisir, retouche de photos, écoute de la musique, visionnage des clips ou des films, etc. 


\section{Des représentations qui convergent mais des demandes contrastées $\rightarrow$}

De manière assez étonnante, les fortes variations observées dans le rapport à la bibliothèque municipale ne font pas obstacle à une convergence de représentations, qui semblent révéler l'ancrage d'habitudes et/ou de perceptions normées, majoritairement légitimées par les jeunes eux-mêmes. Ces perceptions, que l'on peut qualifier de traditionnelles, apparaissent au travers de nombreux thèmes abordés lors des entretiens - appréciation des établissements et de leur offre, perception de l'ambiance, des règles de fonctionnement, image du personnel et du métier, point de vue sur l'utilité des établissements - et peut-être plus encore dans la difficulté à imaginer une bibliothèque idéale. On sera sans doute surpris de constater que ces perceptions sont tout autant le fait d'usagers assidus des établissements que d'adolescents qui ne les fréquentent pas ou plus; elles dépassent aussi très largement les questions d'âge, de genre et de milieu socioculturel, ainsi que l'offre proposée par les établissements. La déclinaison de cette image traditionnelle apparait au travers de nombreux clichés, au titre desquels on peut citer : la bibliothèque reste le lieu du livre et de l'étude, la bibliothèque est un lieu calme et sérieux, les bibliothécaires exercent un métier statique et ennuyeux. Elle n'empêche toutefois pas les adolescents d'exprimer des attentes diversifiées et de revendiquer leur place dans ces lieux.

\section{La maison du livre : un lieu calme et sérieux, légitimement codé}

L'image de la bibliothèque comme lieu du livre est très présente dans les représentations des adolescents rencontrés dans le cadre de cette étude. Peu enclins à utiliser le terme de "médiathèque " (sinon parmi les usagers de la médiathèque José Cabanis de Toulouse), les amateurs de lecture valorisent cette représentation traditionnelle des établissements. Ainsi de 13 à 18 ans, ces lecteurs décrivent une bibliothèque idéale qui resterait « dans la tradition parce qu' une bibliothèque je trouve que c'est assez traditionnel, avec les livres" (Manon, 13 ans), ou encore parce que «ça représente toujours ce qu'on voyait dans les vieux films [avec] des grandes rangées de livres qui renferment tous une histoire différente " (Killian, 18 ans). On repère même chez certains une forme de nostalgie pour ces bibliothèques du passé :

"Dans mon idée la bibliothèque, c'est beaucoup les livres anciens, je trouve c'est un peu mystique, et c'est vrai j'aimerais que ce soit beaucoup plus comme dans les bibliothèques parce que c'est un environnement assez mystérieux. » Gaëlla, 17 ans.

Cette image de la bibliothèque associée aux livres, et plus particulièrement aux romans de fiction, semble largement confortée par des usages que l'on a précédemment pu qualifier eux aussi de "traditionnels " (emprunt de livres et lecture sur place), par la méconnaissance des fonds, collections et supports proposés, ainsi que par le sentiment de satisfaction assez général exprimé par les usagers sur l'offre existante, tout simplement parce qu'il n'imaginent pas trouver d'autres supports ni avoir d'autres pratiques dans les établissements. 
À l'inverse, la représentation de la bibliothèque comme lieu du livre est connotée négativement par tous ceux qui cherchent à se distancier de ce lieu et de son public, ces " autres ", "lecteurs ", "bon élèves " et " intellos ", qui viennent à la bibliothèque parce qu'ils aiment lire ou parce qu'ils «s'embête[nt] un peu (...) pour atterrir là ", tandis que, comme le dit Ruddy, «si on n'aime pas lire, ça c'est autre chose, on a envie de partir (...) parce que là j'ai l'impression que c'est triste, c'est tout gris ». En ce cas, la bibliothèque est à nouveau associée au passé en raison de son affinité avec une activité désuète, la lecture, ainsi que l'explique Fahim :

"Aller à la médiathèque, lire tout ça, on va dire que c'est plus tellement à la mode, entre guillemets, même si de là à dire que lire est une mode, je trouve ça excessif mais bon, on n'est pas forcément attiré. »

Mahera, quant à elle, usager inscrite à Graulhet déplore que ce lien avec le livre dissuade ses camarades de fréquenter la bibliothèque :

«Pour les jeunes maintenant, la médiathèque c'est la lecture [et] la lecture c'est nul... Pourquoi aller à la médiathèque ? C'est la lecture à la base... Plus on avance en âge plus c'est comme ça... »

La bibliothèque apparait alors comme un lieu situé hors du monde de ces adolescents, un endroit où ne vont plus les copains (" Non aucune de mes copines ne va à la médiathèque » "personne autour de moi n’y va, ni les copains, ni ma mère » «mes copains, ça m’étonnerait maintenant qu'ils y aillent ».), investi seulement par les petits ou par les personnes âgées :

«Le point de rencontre des jeunes, c'est plutôt le stade, pas la médiathèque. On se retrouve tous au stade et on va taper un ballon et y a les copains dans les tribunes. C'est plutôt ça que la médiathèque. La médiathèque, ça fait pas intello, mais bon... enfin, ça fait un peu plus pour les personnes âgées que pour les jeunes. Nous, on n'a pas envie d'aller s'enfermer là-dedans un après-midi. » Mickaël, 14 ans.

Contrairement au stade, lieu ouvert, où l'on peut courir, crier - voire réviser ses cours sur la pelouse comme Marguerite, 17 ans, qui prépare un BEP d'hygiène et environnement - la bibliothèque est un monde clos, réglementé et silencieux :

"Oui, ne pas courir, être calme. Moi je suis plutôt... je bouge partout. » Mickaël, 14 ans.

Qu'ils fréquentent ou non les établissements, les adolescents s'accordent pour souligner cette ambiance "calme " des bibliothèques, perçue par les uns comme propice à la détente et/ou à la concentration, par les autres comme " froide ", "super stricte ", voire mortifère, mais dans tous cas comme inhérente au lieu : "c'est calme, normalement une bibliothèque, 
c'est calme " répètent-ils à l'envi. L'ambiance "calme " est connotée très favorablement en particulier par les filles usagers, mais de manière plus générale par tous ceux qui déclarent se "sentir libre " dans les établissements qu'ils fréquentent pour leurs loisirs et la lecture plaisir. Il en est ainsi d'Hélène, 14 ans, qui fréquente assidûment l'espace ados de la bibliothèque centrale d'Auxerre :

"Moi je trouve ça magique... les livres. Pour les livres et pour l'ambiance aussi parce qu'à la bibliothèque il ne faut pas parler fort. C'est mystérieux un peu aussi le fait de ne pas parler fort à la bibliothèque."

On le voit la bibliothèque est, de fait, un lieu calme, " un endroit qui prête à la réflexion " par la présence des « livres nobles " précise Maxime, 17 ans, fervent lecteur de poésie, de théâtre et de romans.

Les usagers inscrits, amateurs de lecture, ne sont pas les seuls à plébisciter ce calme ambiant. En effet, les adolescents qui fréquentent les bibliothèques pour leur travail scolaire apprécient aussi très largement cette atmosphère qu'ils qualifient de favorablement "studieuse ", elle aussi propice à la concentration. À la recherche de ce calme studieux, les plus âgés d'entre eux, le plus souvent rencontrés parmi les usagers non inscrits, se révèlent même les plus critiques à l'égard des établissements où l'absence de cloisons entre les espaces favoriserait une ambiance qu'ils qualifient alors de « trop bruyante ». C'est bien l'ambiance calme, qui fait parfois défaut ailleurs (domicile ou établissement scolaire) et qui contraint à se concentrer sur son travail scolaire qui est recherchée par ces usagers " travailleurs ", jeunes adultes de 17-18 ans.

Le silence des bibliothèques n'est donc pas une contrainte pour une majorité des jeunes usagers interrogés, même si l'enquête quantitative montre qu'un bon tiers d'entre eux trouvent bibliothèque "trop calme " (36\% des 11-14 ans et 30\% des 15-18 ans). Il est par ailleurs tout relatif puisque la circulation entre les différents espaces permet d'alterner entre différents niveaux sonores. Citons en particulier la circulation des espaces de lecture et/ou des salles de travail vers les discothèques ou cédéthèques. Ces espaces dédiés à la musique sont en effet perçus très favorablement, d'autant plus à Graulhet ou à Dinan que la musique est diffusée en fond sonore, que les usagers peuvent écouter un $\mathrm{CD}$ pour le découvrir et que la relation avec son personnel y est semble-t-il plus spontanée. Ces différents éléments confèrent une atmosphère conviviale toute particulière à ces espaces dédiés à la musique et participent à la perception positive attribuée, par contagion, à l'ensemble de l'établissement.

Mais loin de faire l'unanimité parmi les adolescents interviewés, le calme peut être perçu comme "triste ", austère et contraignant, voire tout à fait dissuasif. Désignée par l'absence de bruit et d'ambiance ("sans bruit"; " mais y a pas d'ambiance à la bibliothèque ! "; " c'est la mort!) ou associée $\mathrm{au}$ " silence » religieux ou mortifère (comme à «l'église » ou comme " dans les hôpitaux "), l'ambiance qui se dégage des bibliothèques peut aussi être comparée à l'atmosphère contraignante imposée par le cadre scolaire. On entend ainsi parfois, l'ambiance de la bibliothèque c'est « comme dans la salle 
de classe " ou "pareil que le CDI ", c'est un lieu où finalement " on ne peut pas faire ce qu'on veut " (et en particulier " parler » et " discuter »). Le silence, alors considéré comme strict et austère, est le plus souvent évoqué par les adolescents qui disent ne pas aimer lire ou avoir des difficultés scolaires, et plus généralement par tous ceux qui déclarent préférer travailler avec un fond sonore. Il sera alors désigné comme un "défaut de fabrication » des établissements, dans lesquels les architectes-concepteurs auraient dû créer « un coin silence et coin non-silence (...) avec une ambiance plus vivante ", des " coins » distincts, décrits comme des sortes de «bulles » tolérant différents niveaux sonores.

Cependant, qu'ils l'apprécient ou non, qu'ils soient fréquentants ou non, qu'ils aiment lire ou non, c'est dans leur très grande majorité que les adolescents interviewés considèrent cette ambiance comme allant de soi. À l'image de nombreux non-fréquentants rencontrés dans les différents sites, Rémi, 13 ans, interrogé à Lille évoque le caractère légitime de cette ambiance qu'il ne semble pourtant pas apprécier :

"Lambiance ? Ben c'est comme une bibliothèque, je sais pas, c'est normal... on entend rien. Forcément, comme une bibliothèque (...) c'est normal, c'est une bibliothèque, forcément y a du silence, c'est pas super pour l'ambiance, mais je sais pas c'est une bibliothèque, c'est normal. "

En fait, même si la perception de l'atmosphère tend à se dégrader des fréquentants aux non-fréquentants (passant d'une image axée sur la convivialité et la liberté à une autre, synonyme d'austérité et de contrainte) les différents témoignages révèlent que c'est le caractère sérieux des bibliothèques qui semble conférer la légitimité à cette ambiance - ou à cette absence d'ambiance. Cette image bien ancrée de sérieux attachée aux bibliothèques apparait en particulier dans le refus d'y trouver certains supports tels que les jeux vidéos, ou d'y pratiquer des activités ludiques car la bibliothèque "c'est comme ça ", "c'est pas fait pour jouer ", sinon avec des « jeux pour l'esprit » qui permettraient de «faire progresser sa tête ».

\section{«C'est mystérieux les bibliothécaires » : un métier statique et ennuyeux}

À l'instar de leur méconnaissance des fonds, des supports et des espaces, les adolescents interviewés connaissent très peu les fonctions exercées par le personnel des bibliothèques. Fréquentant ou non les établissements, ils citent le plus souvent des fonctions liées au renseignement (information, conseil et parfois guide - environ $90 \%$ des usagers interrogés par questionnaire à Lille et à Toulouse estiment que les bibliothécaires aident bien) ; ainsi que des tâches liées à l'inscription du public, à l'enregistrement du prêt et des retours, au rangement des documents et à la surveillance. Ponctuellement, certains adolescents évoquent l'organisation et l'animation des activités proposées dans les établissements, en particulier dans le secteur jeunesse. Toutefois, dans la majorité des cas, leur connaissance, assez fragmentaire, des fonctions imparties aux bibliothécaires, se réduit à ce qui est vu, et participe à une image très stéréotypée du métier, voire du personnel lui-même.

Les bibliothécaires apparaissent ainsi, à l'image de leur métier, comme étant un peu mystérieux. Dans ce sens, Hakim, inscrit à la médiathèque centrale de Nanterre se questionne tout autant sur le métier que sur la personnalité des bibliothécaires : 
«La question que je me pose, c'est : est-ce qu'ils restent assis toute la journée au même endroit ou est-ce qu'ils bougent? Je vois bien qu'ils sont souvent assis au même endroit, c'est ça le truc... Ils sont assis derrière leur ordinateur et attendent un renseignement, sinon ils vont aider les usagers ou ils font le retour des documents. C'est mystérieux les bibliothécaires en fait. C'est assez discret. Il y a beaucoup de questions sur ce métier. On ne connaît presque rien d'eux en dehors de ce qu'ils font là. "

Le métier de bibliothécaire est alors souvent considéré comme "pas vraiment fatiguant ", avec un temps de travail limité ("14 heures à 18 heures ") qui permettrait, selon certains, d'exercer une autre activité professionnelle en dehors des bibliothèques; il s'agirait donc d'un métier finalement "pas du tout stressant " pour des « gens qui n'aiment pas se prendre trop la tête ", voire une activité idéale pour les étudiants qui auraient ainsi du temps à consacrer à leurs recherches universitaires.

Mais ce métier semble surtout perçu comme « ennuyeux ", trop calme, à l'image de ce qui se dégage des établissements, et pour lequel il n'y aurait " pas grand-chose à faire ", comme l'explique Thibaut, rencontré à Toulouse :

"Ce métier-là, il est assez bien, mais des fois, si on reste comme ça assis tout le temps, ça va devenir ennuyeux. Des fois, quand ils restent comme ça tout seul pendant des heures devant un écran, on dirait qu'ils s'ennuient. "

Souhaitant souvent exercer un métier " plus dynamique », qui « bouge » demandes contrastées et "avec plus d'actions ", à l'image des activités de loisirs préférés de la plupart d'entre eux, les adolescents insistent sur le caractère statique d'un métier très majoritairement méconnu, si ce n'est par les adolescents qui ont effectué un stage dans des bibliothèques au cours de leur parcours scolaire, souvent en fin de $3^{\text {e }}$.

L'image du métier, le plus souvent associé à l'ennui et au caractère statique et répétitif des tâches à accomplir, jugées peu intéressantes ou gratifiantes, tend à traverser les âges et les sexes ; elle est toutefois assez différenciée selon que les adolescents sont usagers ou non des bibliothèques, et plus largement selon qu'ils sont lecteurs ou non. Cette perception est en effet sensiblement plus positive chez les usagers inscrits, amateurs de lecture, qui associent le métier à la détention d'une bonne culture générale, projetant sur l'institution les valeurs dont ils sont eux-mêmes porteurs et/ou attribuant aux bibliothécaires la mission de donner le goût pour la lecture. À l'inverse, cette aura culturelle dégagée par le personnel des bibliothèques peut être ressentie comme une frontière réelle ou symbolique qui empêcherait toute possibilité de contact, notamment pour les usagers non inscrits, moins familiers des lieux et qui fréquentent les établissements uniquement pour leur travail scolaire. C'est ce que dit explicitement Gaëlla, qui est pourtant inscrite en $1^{\text {re }}$ littéraire : 


\begin{abstract}
«Et puis on a l'impression que c'est des gens... On se fait toujours une idée sur les bibliothécaires, qu'elles ont toujours de la culture, qu'elles savent beaucoup de choses. Et c'est vrai qu'en venant, je me suis dit " peut être qu'on va pouvoir m'aider sur... par exemple mon commentaire de français", et c'est vrai que quand je l'ai vue, je me suis dit "bon, je vais peut être pas demander", (...) je sais pas, et puis parce qu'aussi, elle parlait avec une autre adolescente et elles n'arrêtaient pas de parler livres, et je me suis dit " moi, tous ces livres fantastiques, je ne les ai pas lus, je ne vais pas m'incruster dans la conversation" (...) On a toujours peur de paraitre ridicule face aux gens qui ont plus de culture sur certaines choses que nous. »
\end{abstract}

Ainsi, comme pour celle de l'ambiance, l'image du personnel, et plus généralement du métier de bibliothécaire tend à se dégrader des usagers inscrits, fréquentants les plus réguliers et assidus des établissements, aux non-usagers, en passant par les non-inscrits et les anciens usagers. La représentation évolue, d'un métier de culture et de contacts à un métier statique, où l'on s'ennuie. Conjugués, tous ces éléments concourent alors à l'émergence d'un portait du bibliothécaire ou d'une définition de ce métier qui pourrait être : bibliothécaire, plutôt une femme (pas forcément âgée), aimant particulièrement la lecture et ayant de solides connaissances notamment littéraires, effectuant un travail ennuyeux, le plus souvent assis, dans un lieu très calme et silencieux. Cette image semble donc se substituer au cliché de la femme d'âge mûr, d'allure sévère et taciturne, chignon gris et lunettes, qui a longtemps participé à définir le stéréotype de la bibliothécaire.

\title{
24
}

Des représentations qui convergent mais des demandes contrastées

\section{Des demandes contrastées}

Les stéréotypes toujours ancrés sur les bibliothèques, associés à une intériorisation des codes et des comportements attendus et/ou à une forme de relativisme (" c'est comme ça ", " soit on aime, soit on aime pas ", " c'est une question de choix »), expliquent sans doute le peu d'empressement des adolescents à décrire (ou même seulement à imaginer) une bibliothèque qu'ils considèreraient comme idéale. De sorte que l'absence de critique à l'égard des établissements semble davantage exprimer une forme de consensus mou que relever d'un réel enthousiasme face à l'offre existante. C'est donc le plus souvent à mi-mot que les adolescents rencontrés lors de l'enquête qualitative se risquent à formuler quelques attentes à l'égard de cet univers, implicitement perçu comme peu propice au changement. Ces attentes, qui s'expriment surtout à l'égard d'une offre plus diversifiée et d'une réorganisation des espaces, sont assez contrastées selon les âges mais se rejoignent dans la mesure où elles expriment le souhait d'une reconnaissance : la reconnaissance d'appartenir à un public diversifié (à l'image de la diversité du public adulte), tant dans ses usages que dans ses rapports à la lecture.

Les attentes exprimées par les adolescents interviewés relèvent avant tout des collections qu'ils souhaiteraient plus variées, livres, $\mathrm{BD}$, mangas et $\mathrm{CD}$, 
et actualisées : ils réclament notamment les nouveautés musicales et le suivi dans les séries. Ne formulant presque jamais une réelle insatisfaction par rapport à l'existant, ils semblent toutefois plus critiques à l'égard de l'offre en matière de postes informatiques. Cette critique apparaît quelle que soit l'offre proposée. Des demandes d'accès facilité aux ordinateurs, sans contrainte de réservation préalable, sans limite de temps, et permettant de "surfer " librement sur tous les sites Internet s'expriment également, et cela particulièrement dans les bibliothèques de quartier, comme le révèle l'enquête quantitative par questionnaire.

En matière d'espaces, les jeunes usagers de 11 à 18 ans interrogés par questionnaire à Lille et à Toulouse ont largement revendiqué leur souhait d'un espace dédié : $78 \%$ d'entre eux affirment qu'il est important qu'il existe un espace adolescents dans une bibliothèque. Parmi eux, ce sont les collégiens, de 11 à 14 ans, qui se sont montrés les plus intéressés par l'existence d'un tel espace (le pourcentage s'élève alors à $87 \%$ ). La présence d'un espace dédié aux jeunes permettrait " de se retrouver " entre soi, « de se rencontrer ", pour " discuter » et se distraire, et dans une moindre mesure pour " trouver plus facilement ce que l'on cherche ». Les jeunes de 17-18 ans, eux aussi plutôt favorables à ce type d'espace (pour $64 \%$ d'entre eux), semblent toutefois plus nuancés dans leur réponse. Ne se considérant plus vraiment comme des adolescents, ces jeunes adultes se disent aussi favorables à une libre circulation dans les différents espaces des bibliothèques, pouvant ainsi échanger avec les adultes et «fouiller » dans les documents pour découvrir de nouvelles choses.

On le voit, la question des espaces, et notamment celle d'un espace " ados", se pose différemment selon les âges. Néanmoins, la question de l'organisation spatiale semble surtout renvoyer à la diversité des usages et au niveau de bruit toléré dans chacun d'entre eux : des lieux calmes dédiés à la lecture (avec un fonds spécialisé et diversifié pour les jeunes de leur âge, en particulier pour les plus jeunes) et/ou au travail scolaire (cloisonnés pour les plus âgés, mais qui permettent aussi le travail en groupe), et des lieux dédiés à la détente et aux échanges. La spécialisation des espaces semble donc plus relever de la question des usages que de celle de l'âge : ainsi, quel que soit leur âge, les adolescents rencontrés insistent sur la nécessité d'espaces propices à la concentration, juxtaposés à d'autres espaces plus propices à la convivialité.

Dès lors, la bibliothèque que l'on pourrait peut-être se risquer à qualifier d'idéale pour les adolescents serait plus diversifiée du point de vue des fonds, mais aussi des espaces. Colorée et ouvrant sur l'extérieur par de grandes parois vitrées, cette bibliothèque proposerait également des activités qui s'élargiraient à d'autres champs culturels et sociaux et qui, sans que cela soit clairement dit, favoriseraient leur sociabilité. Quelques adolescents à la fréquentation assidue, et âgés de 16-18 ans, n’hésitent pas non plus à réclamer "des choses qui surprennent", des activités qui bousculeraient un peu l'ordre établi et les idées préconçues sur des établissements qu'ils ne fréquentent pas pour la lecture plaisir. Rappelant que « les jeunes ont leur place partout dans la bibliothèque ", certains jeunes adultes revendiquent " des responsabilités " par le développement d'activités et de service qui leur permettraient de s'impliquer dans l'organisation et le fonctionnement 
des établissements. Cette volonté d'avoir un rôle à part entière dans l'offre proposée aux adolescents apparaît aujourd'hui comme une réelle opportunité : l'opportunité de communiquer avec le(s) public(s) adolescent(s) et de changer l'image attribuée à l'univers des bibliothèques (par l'intermédiaire des aînés, parfois faibles lecteurs) et d'établir les bases d'une relation nouvelle, qui ne reposerait plus seulement sur une méconnaissance et des stéréotypes réciproques.

\section{Des pistes pour rebondir $\rightarrow$}

À l'issue de ces nombreuses heures d'entretien passées avec des jeunes âgés de 11 à 18 ans, les pistes qu'il conviendrait de mettre en œuvre ne manquent pas, même si peu en fait ont été énoncées directement par nos interviewés mêmes. Ces derniers, en effet, sont souvent restés en retrait, voire timorés, quant à l'idée de ce que pourrait être pour eux une «bibliothèque ou une médiathèque idéale ». Appréciation favorable de l'existant, vision convenue de la bibliothèque municipale, ou encore manque d'intérêt pour un sujet qui les indiffère, le thème de la bibliothèque idéale ne semble guère stimuler l'imagination des 11-18 ans rencontrés dans le cadre de cette enquête, et c'est peut-être déjà révélateur en soi. On l'a vu, les perceptions des jeunes sont souvent constituées d'images tenaces (la bibliothèque lieu du livre...) et/ou de ressentis qui peuvent paraître étonnants - les bibliothécaires sont toujours assis(es).... Cependant, certains d'entre eux ont tout de même formulé des propositions qui se révèlent innovantes car elles remettent en question bien des pratiques encore en œuvre dans l'accueil en bibliothèque. Dans tous les cas, ces paroles disent à la fois les forces mais aussi les faiblesses de l'institution, de son image et de celle de son personnel. Des faiblesses qui assombrissent sans aucun doute le devenir de l'institution si elle ne s'adapte pas à la mutation des usages et des besoins. Des forces aussi qui donnent aux bibliothèques tous les atouts nécessaires pour tenter de rebondir et s'affirmer comme le lieu de culture et d'information que bien des établissements centraux incarnent déjà pour les adolescents : un lieu de culture, de loisir et d'information, mais aussi un lieu de proximité et de sociabilité, indispensable à leur éducation, vecteur de lien social, outil de mixité intergénérationnelle et finalement principal lieu emblématique du quartier, pour les annexes, particulièrement appréciées des pré-adolescents.

\section{Première piste : replacer le public jeune au cœur de la réflexion}

La première piste à envisager consiste à replacer ce public jeune, ses pratiques et ses besoins au cœur de la réflexion qui doit présider à la conception de son accueil. Pour cela, le premier pas permettant d'éviter la conception d'une offre trop éloignée des besoins réels (un certain «biblio- ou adultocentrisme ") serait de reconnaittre pleinement les usages diversifiés, et notamment de reconnaître la place à part entière des usagers non inscrits qui fréquentent les établissements soit exclusivement pour leur travail scolaire, soit pour leur plaisir mais très peu pour la lecture romanesque. La bibliothèque est un lieu de découverte et de divertissement avec des 
supports diversifiés. La bibliothèque est un lieu d'apprentissage, tant par son fonds que par les espaces qu'elle met à disposition pour s'informer, se documenter et travailler. La bibliothèque est aussi un lieu de travail, qui offre un cadre propice à l'étude, en dehors de son offre de collection ou de service. Lacceptation des usages que font les jeunes de ces lieux, dans la limite des missions de l'établissement, devrait être un passage obligé si l'on veut que la bibliothèque soit perçue comme un lieu véritablement accueillant (et non pas excluant), ouvert à tous, chaleureux, propice à l'étude comme au divertissement, et ce quel que soit le goût des fréquentants pour la lecture. C'est, sous certains aspects, ce qui a été initié dans les deux réseaux d'Auxerre et de Toulouse, à deux échelles différentes. Auxerre, par un travail d'accompagnement dans la durée auprès d'une population de jeunes adolescents identifiée en tant que telle, a su fidéliser un noyau de jeunes collégiens au sein d'un espace qu'ils se sont complètement appropriés par le biais de collections dédiées, minutieusement sélectionnées, permettant de favoriser les lectures des jeunes, quelles que soit leurs attentes. Toulouse, dans la médiathèque centrale José Cabanis, propose un espace "Intermezzo " : un espace intermédiaire doté de collections hybrides qui plaisent beaucoup aux jeunes mais qui ne leurs sont pas réservées, d'où l'appellation. L'attractivité exercée sur les jeunes est en fait ici suscitée par l'équipement dans son ensemble qui inspire un sentiment de liberté et de modernité chez les jeunes usagers. On notera au passage que ce type de proposition intermédiaire correspond au développement récent d'une offre éditoriale en direction de " jeunes adultes " qui semble avoir tout à fait trouvé son public : le "phénomène " Twillight en est un exemple particulièrement significatif, livresque autant que non livresque.

La bibliothèque n'est pas réductible pour les jeunes à une collection, elle est aussi, voire avant tout, un espace. Or, dans ce sens, ce que nous ont dit les 11-18 ans rencontrés confirme la nécessité de prévoir des espaces séparés selon les usages pour éviter que se télescopent des pratiques incompatibles - en raison du bruit inévitable qu'elle génèrent ou du silence qu'elles nécessitent. Dans certaines bibliothèques idéales évoquées par les jeunes, les espaces seraient éventuellement spécialisés selon les âges, mais surtout distribués selon des usages agencés en fonction des niveaux de bruit autorisés. Ce cloisonnement des espaces repensés par niveau de bruit permet de préserver la mixité des publics, caractéristique qui demeure une des spécificités fortes et reconnues de la bibliothèque publique où se croisent et cohabitent les différentes générations.

\section{Deuxième piste : faire bouger l'offre de manière significative}

La seconde piste est en fait une déclinaison logique de la première. Elle repose sur un principe simple souvent évoqué par certains professionnels : le changement des représentations actuelles des bibliothèques municipales parmi les jeunes ne peut faire l'économie d'une réflexion importante sur l'offre de collection et de services. Si le socle actuel de l'offre en direction des publics jeunes - notamment le socle livresque - ne saurait être brutalement remis en question, une plus grande ouverture vers les pratiques de loisir, 
de culture et de communication des adolescents contemporains s'impose : qu'il s'agisse notamment des périodiques, des films et de la musique dont ils sont friands. Découlant de cette réflexion, viendra alors naturellement la question du classement, de la mise en valeur des ces documents, et pourquoi ne pas parler de la scénographie?

Cette offre, à elle seule, n'est pas de nature à modifier mécaniquement la relation des 11-18 ans avec les bibliothèques municipales, mais elle peut être considérée comme un signe supplémentaire qui leur est spécifiquement adressé. Ne faudrait-il pas également franchir définitivement le pas de la diffusion massive du multimédia et du numérique dans les collections ? Bien des bibliothèques (ou des élus ?) hésitent encore en effet à autoriser la consultation libre et gratuite d'Internet ou tout simplement à augmenter de manière significative le parc informatique des bibliothèques. L'offre de documents numériques et de terminaux dédiés (ordinateurs, consoles de jeux, etc.) est souvent limitée ou bridée dans les établissements de lecture publique alors qu'une écrasante majorité des jeunes fait un usage quotidien de ces supports et contenus numériques au domicile. Tout laisse à penser qu'il est grand temps de bouleverser l'offre et les modalités de sa mise à disposition en faisant du contenu dématérialisé une ressource documentaire comme une autre, en multipliant partout le nombre d'ordinateurs et en favorisant le développement des accès gratuits à Internet qui constituent des ressources comme les autres et pour beaucoup les premières sources d'information des jeunes collégiens et lycéens.

Troisième piste : donner plus de visibilité à la bibliothèque et faire participer les jeunes

La troisième piste consiste à donner plus de visibilité à la bibliothèque et à tenter d'y faire participer activement les jeunes. Au titre des faiblesses, on peut globalement constater une assez grande méconnaissance des différents espaces, des fonds et des ressources offertes par les bibliothèques contemporaines. Peu d'interviewés, en effet, ont une représentation claire de l'offre existante et la quasi-totalité sous-estime l'ensemble des services disponibles. Plusieurs éléments peuvent expliquer cette méconnaissance : la lisibilité difficile de certains établissements proposant une circulation non intuitive entre les espaces (ce qui a pour conséquence de masquer les ressources plutôt que les valoriser), ou le caractère intimidant des bâtiments ou des espaces non balisés... La mise en place d'une meilleure signalétique est un objectif visé par de nombreux professionnels mais qui est encore trop rarement mis en œuvre, surtout en direction des publics adolescents. Les bâtiments ne parlent pas d'eux-mêmes, pas plus de l'extérieur que de l'intérieur. Des formes de médiations spécifiques doivent ainsi être (re)pensées pour favoriser une meilleure connaissance des établissements par les 11-18 ans : documents de communication dédiés sur différents supports, visites d'établissements, etc. Les bibliothèques ne devraient-elles pas également accueillir massivement les jeunes élèves de la classe de troisième à la recherche de stages ? Ces jeunes stagiaires, rencontrés lors de l'étude, sont en effet les seuls à avoir une connaissance précise des espaces, de l'offre proposée et du métier de bibliothécaire. Cette expérience, qu'ils décrivent comme une " découverte ", change considérablement leur 
perception des bibliothèques, au point de les transformer en véritables porteparole d'un univers dont ils étaient parfois très éloignés.

Au-delà de la simple connaissance, certains adolescents rencontrés parmi ceux de 16-17 ans, notamment à Auxerre et à Dinan, émettent le souhait d'être impliqués dans la vie de la bibliothèque : ils demandent à avoir des responsabilités dans l'organisation et le fonctionnement des établissements, ainsi que dans le choix des documents. Il serait ainsi opportun de favoriser la participation des adolescents à des activités leur permettant de s'exprimer. Ce processus peut être conduit par le biais de clubs de lecture, comme cela existe déjà avec plus ou moins de réussite, ou au sein d'instances qui permettraient aux jeunes de participer aux acquisitions, à la définition des programmes d'animation et bien sûr à la conception des espaces leur étant dévolus - en partenariat éventuellement avec d'autres acteurs locaux, culturels ou non. Il est important pour les adolescents d'être reconnus par leurs pairs et par les adultes : les faire participer à la vie de la bibliothèque est une étape vers cette reconnaissance favorisant éventuellement plus tard une réelle appropriation. Ces jeunes " convertis " pourraient à leur tour devenir les meilleurs avocats de la bibliothèque auprès des leurs.

Nous avons déjà évoqué la perception traditionnelle pour ne pas dire passéiste qu'avaient les jeunes des bibliothèques ou médiathèques, surtout quand ils en avaient une fréquentation épisodique ou rare. Cette image constitue certainement la principale faiblesse des bibliothèques. Elles restent encore majoritairement le lieu du livre, à la fois sur le plan des usages et des représentations. Une plus grande communication est nécessaire pour changer cette image. Pour effectuer ce changement, il conviendrait d'engager des actions sur un front assez étendu qui va de la promotion de l'offre de collection et de services à la promotion de l'expertise des professionnels. Certaines bibliothèques, dont la Bibliothèque Francophone de Limoges ou le réseau municipal de Bordeaux se sont lancées récemment dans des campagnes de communication dynamiques sur l'offre de services. Il est difficile de dire si ces campagnes de communication ont des effets directs en termes de fréquentation, d'inscriptions et d'emprunts; elles ont toutefois le mérite de participer à la visibilité et à la connaissance des bibliothèques, et donc de favoriser la construction d'une nouvelle image auprès du public. Toutefois, peu d'entre elles se sont aventurées dans la promotion du métier et des professionnels. Cette piste mériterait peut-être d'être explorée. Il convient à la fois de faire connaître le métier de bibliothécaire, qui participe à l'image de marque des établissements, et surtout de valoriser la compétence des professionnels en multipliant, par exemple, les occasions d'accompagner et de former les jeunes usagers dans leurs recherches documentaires classiques et sur Internet. Il conviendrait également de mettre en avant les compétences informatiques des bibliothécaires pour redorer leur image aux yeux des jeunes pour qui la compétence technique est si valorisante, ou encore de multiplier les formations ou l'animation d'ateliers pour l'animation d'un blog de la bibliothèque, voire d'une web TV, etc. C'est dans cette logique que de nombreuses institutions profitent aujourd'hui des réseaux sociaux du type Facebook ou Myspace pour faire circuler rapidement et facilement des informations auprès des jeunes connectés. Ce type de démarche est susceptible d'avoir un impact sur l'impression d'immobilisme qui se dégage parfois des représentations juvéniles du métier de bibliothécaire. 
Quatrième piste : dynamiser la relation avec les publics jeunes

et les autres partenaires

Sur un plan général, l'adaptation de la formation donnée aux bibliothécaires devrait être de nature à permettre à l'ensemble de la profession d'améliorer sa connaissance et son appréhension des besoins et de la sociabilité particulière des jeunes de 11 à 18 ans. Ceci pourrait conduire éventuellement les professionnels à admettre que les usages que l'on pouvait qualifier de " détournés » (retrouver ses amis, discuter, travailler sur place sans utiliser les ressources de la bibliothèque, etc.) sont aussi légitimes et souhaitables que l'emprunt de livres. C'est une autre occasion pour les bibliothèques de rebondir et d'accueillir de façon satisfaisante ces usagers considérés parfois comme un public particulier sinon difficile. À l'image de ce que proposent parfois certains cycles de formation à l'étranger, on voit mal aujourd'hui comment la formation des bibliothécaires français pourrait faire l'impasse sur une spécialisation "publics adolescents " ou " jeunes adultes " afin que certains personnels soient identifiés dans les établissements comme des référents professionnels pour ces publics ${ }^{19}$. Ici se pose la question de l'accompagnement scolaire des publics juvéniles présents à la bibliothèque et des capacités de médiation d'un personnel spécifiquement formé pour faciliter la maîtrise des supports et des contenus proposés par les bibliothèques (numériques et traditionnels). À la réflexion, ce principe qui pourrait consister à faire " bouger la relation " peut évidemment être également appliqué aux autres partenaires des bibliothèques qui permettent habituellement d'intervenir auprès des jeunes : l'école au premier chef, mais également les vigiles ou le personnel de surveillance dans certains cas. Si la différenciation des espaces permet d'accepter des activités et des publics parfois forts différents quant à leurs comportements et le niveau sonore qu'ils impliquent, alors il devient peut être possible de desserrer l'étau du règlement : non pas déréglementer la bibliothèque mais assouplir certains de ses codes pour faciliter la relation et l'accueil des jeunes.

\section{Conclusion $\rightarrow$}

Ces pistes visant à améliorer les modalités d'accueil et l'offre à destination des jeunes sont déjà appliquées ici ou là. Cependant, si elles étaient appliquées de façon systématique, elles permettraient peut être de modifier le rapport des jeunes à la bibliothèque en changeant en premier lieu l'image qu'ils ont de cette institution. Or, on le sait, et cette enquête l'a encore confirmé, l'image a plus d'impact sur les usages qu'une simple modification de l'offre de services qui ne serait pas relayée par une forte communication.

Tout étant affaire de communication et d'image, il convient donc de bousculer l'inscription de la bibliothèque dans la ville en la plaçant au cœur de toutes les activités et en en faisant un lieu incontournable pour l'accès à la culture, à l'information et à la citoyenneté, mais aussi au divertissement. Pour cela, ne faut-il pas multiplier les partenariats et accepter la participation de professionnels provenant de divers horizons à l'animation des espaces et des activités? Ces perspectives n'auront de réelle efficacité, notamment en 
direction des jeunes les plus éloignés de l'univers des bibliothèques, que si elles s'intègrent dans un projet éducatif, ludique et culturel local à la mesure du quartier ou de la commune.

\section{Notes}

1. Afin de faciliter la lecture, le terme de "bibliothèque " sera privilégié pour parler de manière générale des établissements de lecture publique ; la terminologie propre à chaque site, voire aux établissements, sera toutefois utilisée au cours du développement (bibliothèque ou médiathèque). De la même façon, l'adjectif municipal et l'abréviation BM seront utilisés, sachant cependant que bien des bibliothèques sont maintenant intercommunales : notamment, celle de Graulhet, dans notre panel. $\rightarrow$

2. Olivier Donnat, Les pratiques culturelles des Français à l'ère numérique : enquête 2008, La Documentation française, 2009. $\rightarrow$

3. Sylvie Octoвre, Les loisirs culturels des 6-14 ans, La Documentation française, 2004. $\rightarrow$

4. La dernière édition de l'enquête Pratiques culturelles des Français, par exemple, confirme que les moins de 25 ans restent la tranche d'âge qui compte le plus d'inscrits et de fréquentants, même si leur engagement dans les bibliothèques est moindre que celui de la génération précédente au même âge. Olivier Donnat, op. cit. $\rightarrow$

5. Voir en particulier Claude PoIssenot, Les adolescents et la bibliothèque, Éditions de la Bibliothèque publique d'information/Centre Georges Pompidou, 1997. $\rightarrow$

6. $17 \%$ seulement des Français âgés de plus de 15 ans déclaraient être inscrits dans une bibliothèque municipale au cours des 12 derniers mois en 2008. Olivier Donnat, op. cit. $\rightarrow$

7. Dominique PASQuier, Cultures lycéennes. La tyrannie de la majorité, Autrement (Mutations $\left.\mathrm{n}^{\circ} 235\right), 2005,180$ p. $\rightarrow$

8. Pour nuancer un peu ce constat, rappelons que tous les adolescents interrogés vivaient à proximité d'un réseau de lecture publique. $\rightarrow$

9. Yves Alıx, et Stéphane Wahnich, "Une familiarité distante : Enquête sur le public des bibliothèques municipales parisiennes ", $B B F, 2004, \mathrm{n}^{\circ} 2, \mathrm{p} .62-73 . \rightarrow$

10. Dans Les loisirs culturels des 6-14 ans, Sylvie Octobre présente l'évolution du "degré de familiarité " avec les équipements culturels que développent les jeunes au cours de leur enfance et les variations dans ces trajectoires. Sylvie ОстовRE, op. cit. $\rightarrow$

11. Ces constats vont dans le sens des résultats des travaux menés par Claude Poissenot et de Sylvie Octoвre qui ont montré qu'il est un âge où la présence des parents devient embarrassante car infantilisante, même si les jeunes ne le disent pas ouvertement dans les entretiens. $\rightarrow$ 12. Ces pratiques restent toujours majoritaires dans les bibliothèques françaises ainsi qu'il apparaît dans l'enquête de Bruno MARESCA, Les bibliothèques municipales en France après le tournant Internet, Éditions de la Bibliothèque publique d'information/Centre Pompidou, 2007, page 66 sq. $\rightarrow$

13. Mariangela RoselLI, «Usagers et usages devant une offre de lecture publique libre : parcours d'acculturation et formes d'appropriation lettrées ", Sociétés contemporaines, n $64,2006 / 4$. $\rightarrow$ 14. Notons que la taille des établissements semble encore compliquer cette libre circulation puisque, selon les résultats de l'enquête quantitative menée à Lille et Toulouse, près de $18 \%$ des adolescents interrogés déclarent avoir du mal à se repérer dans les bibliothèques qu'ils fréquentent, pourcentage qui s'élève à $25 \%$ à la médiathèque José Cabanis. $\rightarrow$

15. Jean Levy, Ségolène Petite, Laurence Le Douardin, Les étudiants de la Médiathèque.

En ligne : http://www.abf.asso.fr/IMG/pdf/douarin.pdf $\rightarrow$

16. Ces différents arguments font écho à l'étude réalisée par Sherry J. Cook qui montre que les jeunes sont tous favorables à la fréquentation de la bibliothèque, mais que la plupart du temps ils n'y vont pas parce qu'ils " n’y pensent pas " tout simplement. Sherry J. CoOK, R. Stephen PARKer, Charles E. Pettijhon, "Les jeunes ados et la bibliothèque publique : une enquête américaine ", trad. Cécile Touitou, $B B F, 2008, n^{\circ}$ 6, p. 81-86. $\rightarrow$

17. Dans un rapport commandé par Scholastic (2008 Kids \& Family Reading Report: Reading in the 21st Century: Turning the Page with Technology), on apprend que pour $89 \%$ des jeunes, les meilleurs livres sont ceux qu'ils ont trouvé par eux-mêmes (enquête menée auprès de 501 enfants américains âgés de 5 à 17 ans). $\rightarrow$

18. Voir aussi : Yann Nicolas, "Le téléchargement sur les réseaux de pair à pair ", Développement culturel, DEPS, $\mathrm{n}^{\circ}$ 148, juin 2005, qui en 2005 constatait que "les jeunes sont les plus gros utilisateurs des réseaux de pair à pair. Près de la moitié des 15-24 ans ont déclaré avoir téléchargé au moins un fichier contre un quart des internautes âgés de 25 ans et plus ». $\rightarrow$

19. Le recrutement des personnels destinés à travailler auprès des adolescents et jeunes adultes est évidemment à prendre également en considération ici : tant en ce qui concerne l'âge que le genre du personnel recruté. $\rightarrow$ 


\section{Annexe $\rightarrow$}

\section{Annexe quantitative}

Dans le cadre de l'enquête portant sur les 11-18 ans et les bibliothèques municipales ${ }^{1}$, un questionnaire autoadministré a été diffusé au cours de l'année 2008 dans les réseaux de Lille et Toulouse, c'est-à-dire dans les bibliothèques centrales et dans les annexes de ces deux réseaux².

1228 questionnaires utiles ont été produits dans le cadre de cette enquête - 697 à Toulouse et 531 à Lille -, par des fréquentants inscrits et des fréquentants non inscrits ( $77 \%$ d'inscrits en moyenne et $33 \%$ d'usagers non inscrits). On compte en moyenne $66,5 \%$ de filles et $33,5 \%$ de garçons ; $53 \%$ des personnes interrogées ont entre 11 et 14 ans et $47 \%$ ont entre 15 et 18 ans.

\begin{tabular}{|l|c|}
\hline $\begin{array}{l}\text { La première fois que tu es allé dans une bibliothèque, } \\
\text { quel âge avais-tu? }\end{array}$ & Moyenne \\
\hline Moins de 6 ans & $34 \%$ \\
\hline Entre 6 et 10 ans & $34 \%$ \\
\hline Entre 11 et 15 ans & $16,5 \%$ \\
\hline Plus de 15 ans & $6 \%$ \\
\hline Je ne me souviens pas & $10 \%$ \\
\hline TOTAL & $100 \%$ \\
\hline
\end{tabular}

(Ensemble des personnes interrogées)

\begin{tabular}{|l|c|c|c|}
\hline Tu fréquentes une autre bibliothèque ? & $\mathbf{1 1 - 1 4}$ ans & $\mathbf{1 5 - 1 8}$ ans & Moyenne \\
\hline Une autre bibliothèque municipale & $23 \%$ & $20 \%$ & $22 \%$ \\
\hline Le bibliobus de la bibliothèque & $6 \%$ & $4 \%$ & $5 \%$ \\
\hline Une bibliothèque d'une autre ville & $8 \%$ & $14 \%$ & $11 \%$ \\
\hline Un CDI & $44 \%$ & $30 \%$ & $37,5 \%$ \\
\hline Aucune autre bibliothèque & $27 \%$ & $25 \%$ & $26 \%$ \\
\hline
\end{tabular}

Ensemble des personnes interrogées; plusieurs réponses possibles) 


\begin{tabular}{|l|c|c|c|}
\hline $\begin{array}{l}\text { Quand tu empruntes, le plus souvent tu } \\
\text { empruntes quoi ? }\end{array}$ & $\begin{array}{c}\mathbf{1 1 - 1 4} \\
\text { ans }\end{array}$ & $\begin{array}{c}\mathbf{1 5 - 1 8} \\
\text { ans }\end{array}$ & Moyenne \\
\hline Je n'emprunte pas & $10 \%$ & $25 \%$ & $16,5 \%$ \\
\hline Des romans (hors BD) & $59 \%$ & $47 \%$ & $53 \%$ \\
\hline Des livres documentaires & $21,5 \%$ & $28 \%$ & $24 \%$ \\
\hline Des BD (hors Mangas) & $50 \%$ & $17 \%$ & $35 \%$ \\
\hline Des Mangas & $31 \%$ & $16 \%$ & $24 \%$ \\
\hline Des magazines, revues & $23 \%$ & $13 \%$ & $19 \%$ \\
\hline Des CD musique & $20 \%$ & $21 \%$ & $20 \%$ \\
\hline Des CDROMS, logiciels d'études et de jeux & $9,5 \%$ & $3 \%$ & $6,5 \%$ \\
\hline Des films vidéo ou DVD & $27 \%$ & $26 \%$ & $27 \%$ \\
\hline Autre & $3 \%$ & $2 \%$ & $8 \%$ \\
\hline
\end{tabular}

(Usagers inscrits seulement; plusieurs réponses possibles)

\begin{tabular}{|l|c|c|c|}
\hline $\begin{array}{l}\text { Parmi les espaces suivants, s'ils existent } \\
\text { dans cette bibliothèque, lesquels préfères-tu ? }\end{array}$ & $\begin{array}{c}\mathbf{1 1 - 1 4} \\
\text { ans }\end{array}$ & $\begin{array}{c}\mathbf{1 5 - 1 8} \\
\text { ans }\end{array}$ & Moyenne \\
\cline { 1 - 4 } Pas de préférence & $20 \%$ & $23 \%$ & $21 \%$ \\
\hline Espace jeunesse & $36 \%$ & $13 \%$ & $26 \%$ \\
\hline Espace musique & $22 \%$ & $27 \%$ & $24 \%$ \\
\hline Espace BD & $41 \%$ & $17 \%$ & $30 \%$ \\
\hline Espace revue, presse & $17 \%$ & $15 \%$ & $16 \%$ \\
\hline Espace de travail & $14,5 \%$ & $37 \%$ & $24 \%$ \\
\hline Espace multimédias lordinateurs, Internet) & $30 \%$ & $23 \%$ & $27 \%$ \\
\hline
\end{tabular}

(Ensemble des personnes interrogées; plusieurs réponses possibles) 


\begin{tabular}{|l|c|c|c|}
\hline $\begin{array}{l}\text { Une bibliothèque/médiathèque, } \\
\text { c'est plutôt un lieu... }\end{array}$ & $\begin{array}{c}11-14 \\
\text { ans }\end{array}$ & $\begin{array}{c}15-18 \\
\text { ans }\end{array}$ & Moyenne \\
\hline Pour les petits, les enfants & $18 \%$ & $14 \%$ & $16 \%$ \\
\hline Pour les intellos & $12 \%$ & $9 \%$ & $11 \%$ \\
\hline Pour les retraités, les personnes âgées & $10,5 \%$ & $8 \%$ & $9 \%$ \\
\hline Pour les filles & $12 \%$ & $5 \%$ & $9 \%$ \\
\hline $\begin{array}{l}\text { Pour les jeunes qui n'ont pas de place pour } \\
\text { travailler chez eux }\end{array}$ & $18 \%$ & $24 \%$ & $21 \%$ \\
\hline Pour les jeunes qui n'ont pas Internet & $20 \%$ & $21 \%$ & $21 \%$ \\
\hline Pour les jeunes qui font des études & $19 \%$ & $27 \%$ & $23 \%$ \\
\hline Pour tout le monde & $90,5 \%$ & $84 \%$ & $87 \%$ \\
\hline
\end{tabular}

(Ensemble des personnes interrogées; plusieurs réponses possibles)

\begin{tabular}{|l|c|c|c|}
\hline $\begin{array}{l}\text { Une bibliothèque/médiathèque, } \\
\text { c'est un espace surtout pour... }\end{array}$ & $\mathbf{1 1 - 1 4}$ ans & $\mathbf{1 5 - 1 8}$ ans & Moyenne \\
\hline Pour lire & $86 \%$ & $67 \%$ & $77,5 \%$ \\
\hline Pour rencontrer d'autres gens & $20 \%$ & $14,5 \%$ & $18 \%$ \\
\hline Pour se détendre, passer un moment & $50 \%$ & $34 \%$ & $43 \%$ \\
\hline Pour travailler dans le calme & $56 \%$ & $74,5 \%$ & $64 \%$ \\
\hline Pour travailler à plusieurs & $32,5 \%$ & $43 \%$ & $37 \%$ \\
\hline Pour se faire aider dans les recherches & $44 \%$ & $38 \%$ & $41 \%$ \\
\hline Autre & $5 \%$ & $3 \%$ & $4 \%$ \\
\hline
\end{tabular}

(Ensemble des personnes interrogées; plusieurs réponses possibles) 


\begin{tabular}{|l|c|c|c|}
\hline $\begin{array}{l}\text { Quand tu as du temps libre, tes activités } \\
\text { préférées sont... }\end{array}$ & $\begin{array}{c}11-14 \\
\text { ans }\end{array}$ & $\begin{array}{c}15-18 \\
\text { ans }\end{array}$ & Moyenne \\
\hline Etre avec des copains & $63 \%$ & $74 \%$ & $68 \%$ \\
\hline Faire de la musique, dessiner, peindre & $33 \%$ & $32 \%$ & $32,5 \%$ \\
\hline $\begin{array}{l}\text { Faire du shopping, se promener au } \\
\text { centre commercial }\end{array}$ & $41 \%$ & $46 \%$ & $43 \%$ \\
\hline Faire du sport ou de la danse & $48 \%$ & $47 \%$ & $47 \%$ \\
\hline Regarder la télévision, des DVD & $52 \%$ & $43 \%$ & $47 \%$ \\
\hline Ecouter de la musique & $55 \%$ & $64,5 \%$ & $59 \%$ \\
\hline Utiliser l'ordinateur & $61 \%$ & $53 \%$ & $57 \%$ \\
\hline Rester à la maison & $32,5 \%$ & $26 \%$ & $30 \%$ \\
\hline Lire & $60 \%$ & $45 \%$ & $53 \%$ \\
\hline Etre dehors & $44 \%$ & $38 \%$ & $41 \%$ \\
\hline Autre & $6 \%$ & $3 \%$ & $5 \%$ \\
\hline
\end{tabular}

(Ensemble des personnes interrogées ; plusieurs réponses possibles)

\begin{tabular}{|l|c|c|c|}
\hline $\begin{array}{l}\text { Quand tu as une recherche à } \\
\text { faire pour ton travail scolaire, } \\
\text { d'abord tu... }\end{array}$ & $\mathbf{1 1 - 1 4}$ ans & $\mathbf{1 5 - 1 8}$ ans & Moyenne \\
\hline $\begin{array}{l}\text { Cherches dans les livres qui sont } \\
\text { chez toi }\end{array}$ & $41 \%$ & $38 \%$ & $40 \%$ \\
\hline $\begin{array}{l}\text { Demandes à tes parents, } \\
\text { ou frères/soeurs }\end{array}$ & $40 \%$ & $21 \%$ & $32 \%$ \\
\hline Demandes à des copains & $19 \%$ & $13 \%$ & $16 \%$ \\
\hline Vas à la bibliothèque & $45 \%$ & $42 \%$ & $43 \%$ \\
\hline Demandes aux bibliothécaires & $16 \%$ & $10 \%$ & $14 \%$ \\
\hline Vas au CDI & $29 \%$ & $20 \%$ & $25 \%$ \\
\hline Utilises Internet & $69 \%$ & $78 \%$ & $73 \%$ \\
\hline Autre & $2 \%$ & $2 \%$ & $2 \%$ \\
\hline
\end{tabular}

(Ensemble des personnes interrogées; plusieurs réponses possibles) 


\begin{tabular}{|l|c|c|c|}
\hline Qu'aimes-tu lire ? & $\mathbf{1 1 - 1 4}$ ans & $\mathbf{1 5 - 1 8}$ ans & Moyenne \\
\hline Je ne lis pas & $6 \%$ & $5 \%$ & $6 \%$ \\
\hline Des journaux, magazines et revues & $37 \%$ & $56 \%$ & $46 \%$ \\
\hline Des romans policiers ou d'espionnage & $32 \%$ & $27 \%$ & $30 \%$ \\
\hline $\begin{array}{l}\text { Des séries (comme Harry Potter, } \\
\text { Orphelins Baudelaire, etc.) }\end{array}$ & $40 \%$ & $30 \%$ & $35 \%$ \\
\hline $\begin{array}{l}\text { Des romans de science-fiction ou } \\
\text { fantastiques }\end{array}$ & $34,5 \%$ & $30 \%$ & $32 \%$ \\
\hline D'autres romans & $25 \%$ & $36 \%$ & $30 \%$ \\
\hline $\begin{array}{l}\text { Des romans qui parlent des jeunes de } \\
\text { ton âge }\end{array}$ & $41 \%$ & $28 \%$ & $35 \%$ \\
\hline Des livres documentaires & $22 \%$ & $22 \%$ & $22 \%$ \\
\hline Des récits d'aventure ou de voyage & $21 \%$ & $19 \%$ & $20 \%$ \\
\hline Des livres de poésie ou de théâtre & $10 \%$ & $19 \%$ & $14 \%$ \\
\hline Des BD & $62 \%$ & $30 \%$ & $47 \%$ \\
\hline Des mangas & $42 \%$ & $22 \%$ & $33 \%$ \\
\hline Autre & $4 \%$ & $6 \%$ & $5 \%$ \\
\hline
\end{tabular}

(Ensemble des personnes interrogées; plusieurs réponses possibles)

36

Annexe quantitative

\begin{tabular}{|l|c|c|c|}
\hline $\begin{array}{l}\text { D'où provient le dernier livre } \\
\text { - ou la dernière BD - que tu as lu ? }\end{array}$ & $\mathbf{1 1 - 1 4}$ ans & $\mathbf{1 5 - 1 8}$ ans & Moyenne \\
\hline Je ne me souviens plus & $10 \%$ & $11,5 \%$ & $11 \%$ \\
\hline De la maison & $14 \%$ & $12 \%$ & $13 \%$ \\
\hline D'un copain & $6 \%$ & $8 \%$ & $6,5 \%$ \\
\hline Je l'ai acheté & $17 \%$ & $37 \%$ & $26 \%$ \\
\hline C'est un cadeau & $5 \%$ & $4 \%$ & $5 \%$ \\
\hline De la bibliothèque municipale & $38 \%$ & $21 \%$ & $31 \%$ \\
\hline Du CDI & $7 \%$ & $3,5 \%$ & $6 \%$ \\
\hline Autre & $3 \%$ & $3 \%$ & $3 \%$ \\
\hline TOTAL & $100 \%$ & $100 \%$ & $100 \%$ \\
\hline
\end{tabular}

(Ensemble des personnes interrogées) 


\begin{tabular}{|l|c|c|c|}
\hline Dirais-tu que lire des livres... & $\mathbf{1 1 - 1 4}$ ans & $\mathbf{1 5 - 1 8}$ ans & Moyenne \\
\hline Permet de rêver, de s'évader & $51 \%$ & $63 \%$ & $56 \%$ \\
\hline Est nécessaire pour apprendre & $36 \%$ & $54 \%$ & $44 \%$ \\
\hline Est un plaisir & $71 \%$ & $67 \%$ & $69 \%$ \\
\hline Demande du temps & $16 \%$ & $30,5 \%$ & $22,5 \%$ \\
\hline Demande un effort & $10,5 \%$ & $10 \%$ & $10 \%$ \\
\hline M'ennuie & $7 \%$ & $4 \%$ & $5 \%$ \\
\hline $\begin{array}{l}\text { Avec Internet, il n'y a pas besoin } \\
\text { de lire des livres }\end{array}$ & $8 \%$ & $3,5 \%$ & $6 \%$ \\
\hline Je n'ai pas d'avis & $7 \%$ & $2 \%$ & $5 \%$ \\
\hline
\end{tabular}

(Ensemble des personnes interrogées; plusieurs réponses possibles)

1. Enquête réalisée par Tosca-Consultants et BS-Consultants à la demande de la Direction du livre et de la lecture du ministère de la Culture et de la Communication et pilotée par le service Études et recherche de la Bibliothèque publique d'information du Centre Pompidou. $\rightarrow$

2. Pendant une semaine complète d'ouverture, le questionnaire était systématiquement distribué aux personnes âgées de 11 à 18 ans entrant dans les bibliothèques sélectionnées pour l'opération. $\rightarrow$ 